\title{
APOLLO2 YEAR 2010
}

\author{
RICHARD SANCHEZ", , IGOR ZMIJAREVIC ${ }^{1}$, M. COSTE-DELCLAUX ${ }^{1}$, EMILIANO MASIELLO ${ }^{1}$, \\ SIMONE SANTANDREA ${ }^{1}$, EMANUELE MARTINOLLI ${ }^{2}$, \\ LAURENCE VILLATE ${ }^{2}$, NADINE SCHWARTZ ${ }^{3}$ and NATHALIE GULER ${ }^{3}$ \\ ${ }^{1}$ Commissariat à l'Energie Atomique et aux Energies Alternatives \\ Direction de l'Energie Nucléaire \\ Service d'Etudes de Réacteurs et de Mathématiques Appliquées \\ CEA de Saclay, DM2S/SERMA \\ 91191 Gif-sur-Yvette cedex, France \\ ${ }^{2}$ AREVA Engineering \& Projects \\ Tour AREVA, Pl. J. Millier \\ 92084 Paris La Défense, France \\ ${ }^{3} \mathrm{EDF}$ R\&D \\ 1, Av. du Général de Gaulle \\ F92141 Clamart CEDEX, France \\ *Corresponding author. E-mail : richard.sanchez@cea.fr
}

Received July 24, 2010

This paper presents the most important developments implemented in the APOLLO2 spectral code since its last general presentation at the $1999 \mathrm{M} \& \mathrm{C}$ conference in Madrid. APOLLO2 has been provided with new capabilities in the domain of cross section self-shielding, including mixture effects and transfer matrix self-shielding, new or improved flux solvers (CPM for RZ geometry, heterogeneous cells for short MOC and the linear-surface scheme for long MOC), improved acceleration techniques $\left(\mathrm{DP}_{1}\right)$, that are also applied to thermal and external iterations, and a number of sophisticated modules and tools to help user calculations. The method of characteristics, which took over the collision probability method as the main flux solver of the code, allows for whole core two-dimensional heterogeneous calculations. A flux reconstruction technique leads to fast albeit accurate solutions used for industrial applications. The APOLLO2 code has been integrated (APOLLO2-A) within the ARCADIA ${ }^{\circledR}$ reactor code system of AREVA as cross section generator for PWR and BWR fuel assemblies. APOLLO2 is also extensively used by Electricité de France within its reactor calculation chain. A number of numerical examples are presented to illustrate APOLLO2 accuracy by comparison to Monte Carlo reference calculations. Results of the validation program are compared to the measured values on power plants and critical experiments.

KEYWORDS : APOLLO2, Self-shielding, Flux Reconstruction, Method of Characteristics, Industrial Application

\section{INTRODUCTION}

The APOLLO2 spectral transport code, developed at the Commissariat à l'Energie Atomique et aux Energies Alternatives (CEA) with financial support from AREVA and EDF, is widely used for cross section generation and direct transport calculations, including a large range of applications in reactor physics, criticality safety studies and fuel cycle analysis. Its utilization covers $R \& D$ analysis, interpretation of reactor experiments and industrial applications. The code is an integrated component for multigroup cross section generation of other CEA and third-party industrial software packages and it is also used for benchmarking and educational activities.

APOLLO2 is used for routine as well as for reference transport calculations. It is the main tool used for the design and analysis of all the CEA reactors and experimental facilities. [1] The code has been and is being intensively used for the conception of the new CEA experimental reactor JHR. [2, 3] In particular, the unique capability of APOLLO2 to compute curved fuel plates shaped along circle involutes [4] has been used for the core cycle analysis of the High Flux Reactor of the Laue Langevin Institute. [5] APOLLO2 provides self-shielded cross sections and neutron spectra for the DARWIN $[6,7]$ code system for fuel cycle studies and it is also an integrated component of the French Institute for Radiation Protection and Nuclear Safety (IRSN) criticality package CRISTAL [8] for safety assessments, where the self-shielding procedures and the discrete ordinates and collision probabilities solvers of APOLLO2 are put to use to generate homogenized cross sections for multigroup Monte Carlo calculations. The latest version of 
the package uses also the depletion module of APOLLO2 via the DARWIN package for burnup credit.

The APOLLO2 code has been integrated (APOLLO2A) within the ARCADIA ${ }^{(B)}$ reactor code system [9] of AREVA as cross section generator for PWR and BWR fuel assemblies. It is also extensively used by the French utility Electricité de France (EDF) for reactor physics analysis and multigroup library generator, [10] and is a major component of EDF's core calculation chain providing input data to the core code. APOLLO2 is used in the European NURESIM/NURISP platform for nuclear reactor simulation [11] as the main tool for cross section generation and for two-dimensional detailed whole core calculations. It is also a main component of the threedimensional coupled core-plant system for accidental reactor transient simulation HEMERA. [12]

The current version of the code (APOLLO2 2.8) uses the external cross section library APOLIB based mainly on the most recent JEFF nuclear data evaluation. A continuous effort is done to validate newly evaluated nuclear data, such as JEFF-3.1.1. [13] Accurate calculation schemes have been defined: a two-level scheme, based on collision probabilities (CP) with the XMAS [14] 172-groups energy mesh followed by a 20 -groups discrete-ordinates finite-differences or transverse nodal calculation, was recommended with the previous APOLLO2.5 package, [10] while a full MOC scheme with the 281-group SHEM [15] energy mesh has been adopted for the latest APOLLO2.8 package. [16, 17] APOLLO2 calculations of the various neutronics parameters are validated against LWR targeted experiments [18, 19] performed in the EOLE and MINERVE experimental reactors located at CEA-Cadarache. [20, 21] Fuel inventory prediction is validated against Post-Irradiation Experiments carried out in French PWR and German BWR assemblies. [22] APOLLO2 itself is consistently verified [23] with the CEA continuous-energy Monte Carlo code TRIPOLI-4, $[24,25]$ whose cross sections are generated with the same nuclear-data processing tool GALILEE. [26]

The APOLLO2 multigroup transport code was written from scratch incorporating and extending the capabilities of the original APOLLO code. [27, 28] The code was written in FORTRAN 77 and was organized in a modular structure thanks to three auxiliary in-house software packages, [29, 30] which allow for data structuring, dynamic memory management and the use of a user's command language [31] that facilitates the organization of the input stream with the help of user's procedure libraries. Also, new developments are tested on multiple platforms against a problem data base which is regularly updated.

The general characteristics of the first version of the code were presented twenty two years ago [32] and an update was published twelve years latter. [33] In the meantime a large number of publications and presentations in international meetings have illustrated example applications and the new methodologies incorporated in the code. Detailed presentations of APOLLO2 have also been the object of three workshops. [34 - 36]

The purpose of this paper is to present the most important developments that have been incorporated in the code since the last general presentation at the Madrid international meeting. [33] Besides the external multigroup data and the verification and qualification work, the basic ingredients of a spectral code are physical models and transport solvers. The formers consist mainly of homogenization models: cross section self-shielding techniques [37, 38] to compute problem-dependent multigroup data and leakage models [39, 40] and equivalence methods $[41,42]$ to construct the multi parameterized libraries that are passed to the core code for core kinetic and depletion calculations. Moreover, the code can treat stochastic dispersions of grains in homogeneous matrices by the double heterogeneity technique for both the collision probability method $[43,44]$ and the method of long characteristics, $[45,46]$ and has the capability to carry out leakage calculations for mutually interacting assemblies. [32] APOLLO2 flux solvers are based either on the collision probability method (CPM) - full CPM, interface-current techniques (ICT) [47] and simplified ICT (multicell methods) [32] - or on different spatial discretizations of the discrete ordinates form of the transport equation comprising finite differences, transversenodal and short and long characteristics (MOC) methods. $[48,4]$ With the exception of the integro-differential transport (IDT) module, [48] which has three-dimensional capabilities, all APOLLO2 solvers cover the one- and twodimensional geometries. Besides these main components there are a number of tools to perform depletion calculations, perturbation reactivity analysis [49] and first-order perturbation analysis, on-the-fly tabulations of mathematical functions, constructors for geometries and internal objects used by the code, and many more.

In this paper we focus on a presentation of the most relevant techniques that have been lately added to the code. In the next section we briefly describe the modular structure of the code. In Section 3 we briefly review the external cross section library of APOLLO2, while in the following section we present the new self-shielding models. A flux reconstruction technique that speeds up the depletion calculations is presented in Section 5. Recent flux solver developments are presented in the two following sections. Section 6 discusses an RZ collision probability method, while Section 7 deals with recent developments for the short and long characteristic methods. Section 8 illustrates the capability of APOLLO2 to carry out two-dimensional whole core transport calculations, Section 9 gives a brief summary of the most relevant additional features and Sections 10 and 11 give a description of the use of APOLLO2 in AREVA's ARCADIA ${ }^{\circledR}$ reactor codes system and in EDF's industrial calculation schemes, respectively. We conclude with some comments and a short discussion in Section 12. 


\section{MODULAR STRUCTURE}

The modularity of APOLLO2 is based on the interplay between two type of components: modules and objects. The code is composed of a number of independent modules, each of which performs a specific task (construction of a geometry, self-shielding, computation of multigroup collision matrices, performing an external iteration, etc.) and can be viewed as a symbolic operator that acts on input data to create output data. With the exception of the original user's input data, all other data processed by the modules, be it input or output, is organized into typified structures which can be thought of as objects (geometry, isotopic library, collision probability matrices, multigroup flux, etc.) and are identified by user's given names. In this way, the user can utilize the macrolanguage to define its own chain of calculations as a sequential calling of modules where output structures from previous modules are used as input data in subsequent modules. The macrolanguage supports also conditional IF's and LOOP's, allowing the user to perform sophisticated calculations such as computations of critical parameters or depletion calculations involving structural or power changes. An ARCHIVE module allows for permanent data storage, so that calculation results as well as calculation procedures can be stored for future use.

Because APOLLO2 is a modular code, the possible combination of modules to effectuate a given calculation can vary according to user's aims. Therefore, there are no general flow charts. However, by their very nature transport calculations require a sequence of operations comprising non interchangeable steps. For instance, to compute a flux one needs to define a geometry, to assign to it materials (which have been previously made up from references to an external microscopic isotopic multigroup library), to compute microscopic self-shielded cross sections, to compute an internal macroscopic library and, finally, to utilize a multi-group iterator and a flux solver (with a specific numerical method to solve the one-group transport equation). It is thus possible, without getting into user's specific details, to present 'generic' flow charts for typical calculations, and these can be helpful for a better understanding of how the code may be used. As an example, we give in Fig. 1 flow charts for some of the compound operators that may be required for a typical assembly burnup calculation.

An assembly depletion calculation consists of a repeated chaining of two operators. The time-step (TS) operator updates the isotopic concentrations after one time step and outputs these concentrations and the corresponding scalar fluxes for all depletion regions defined by the user. At this point a post-processing (PP) operator homogenizes the concentrations over a set of macro regions and homogenizes and collapses fluxes and reaction rates over a set of macro regions and macro groups. The PP operator uses these data to construct a external parameterized library for later use in a threedimensional diffusion or simplified $\mathrm{P}_{N}$ core calculation. The homogenization step may be done by using a nonlinear equivalence procedure or by direct flux weighting and calculation of flux discontinuity coefficients. [42] These two compound operators are applied as many times as time steps have been defined by the user.

In turn, the TS operator comprises several other operators: The first time through the predictor $(\mathrm{P})$ operator extrapolates over the time step flux and reaction rates from values obtained in previous time steps or from initial values. These extrapolations are used by the isotopicdepletion module to compute isotopic concentrations at the end of the time step, at which point a new flux calculation is done, yielding the flux and the reaction rates. Finally, these flux and reaction rates are compared with the values predicted by the extrapolation and, if the error is greater than a user's defined value, the procedure enters the 'corrector' phase, where the last flux and reaction rates are now used by the $\mathrm{P}$ operator to interpolate the flux within the time step. The time step is then recalculated and the cycle is repeated until convergence is achieved or the number of predictor-corrector sweeps exceed a pre defined value. Note that a different implementation of the TS operator may consist of bypassing the 'corrector' phase and decide instead of halving the length of the time step.

The flux-calculation compound operator solves a source or critical multi-group problem. It may contain a

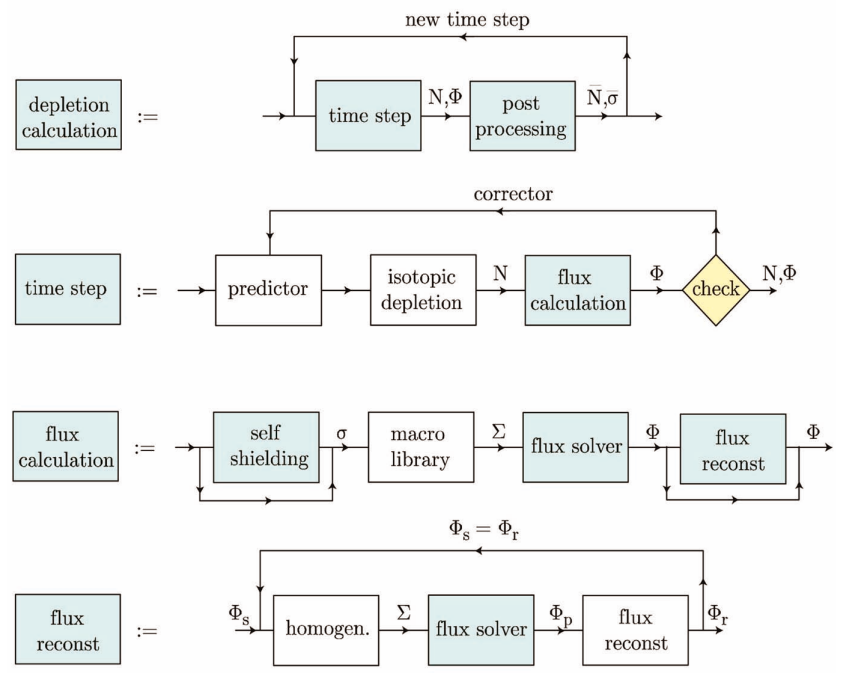

Fig. 1. Generic Flow Charts for Compound Operators Corresponding to Typical Transport Calculations for Reactor Analysis. From Top to Bottom: Depletion Calculation, Time Step, Flux Calculation and Flux Reconstruction Operators. Input Data Have Not Been Specified. White Boxes Correspond to APOLLO2 Modules (single operators). Feed Back and Skip Forward Paths are Controlled by User's Specifications 
first step where multigroup self-shielded cross sections are evaluated and a last step where a flux-reconstruction (FR) operator is used to compute the final flux, but definitely it must comprise the evaluation of an internal multigroup library of macroscopic cross sections that is then used to compute the flux via a flux-solver (FS) operator. The latter can be also a compound operator, comprising an initialization phase and an iterative application of an outer-iteration, an error-check and a fission-source operators or, as it is the case for calculations based on the method of characteristics, the use of a special APOLLO2 module that directly computes the multigroup flux.

The flow-chart in Fig. 2 shows a diagram for the compound self-shielding operator. As detailed in Section 5 , a reconstructed flux $\phi_{r}$, detailed in energy and space, can be obtained from the product of two fluxes: a shape flux $\phi_{s}$, which accounts for a detailed energy description, and a pivot flux $\phi_{p}$ obtained from a precise spatial treatment with a coarser energy mesh. The FR operator consists of an iterative application of three sequential operations. The first operator uses the input shape flux to homogenize a set of macroscopic cross sections ('homogenize' is used here to denote either space homogenization or energy collapsing or both) which are used by the FS operator to compute the pivot flux; then the two fluxes are combined in a simple flux-reconstruction operator to produce the reconstructed flux. Depending on user's aims this last flux can be re inserted, one or more times, as a shape flux to repeat the procedure where, at each pass, different geometry descriptions, homogenization domains and/or flux solvers can be introduced.

This technique allows for fast solutions for problems where the precise distribution is needed in geometrically complicated domains. For example, in the BWR assembly calculations one can treat the control blade as a separate domain with the detailed description of the control elements and channel box structures, while the active zone is

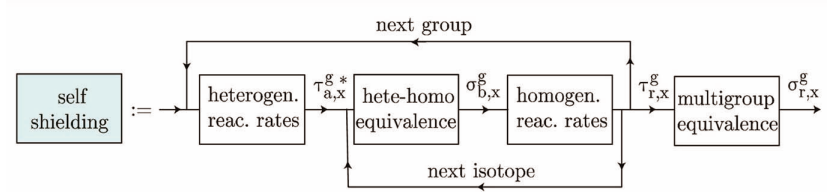

Fig. 2. Schematics of a Self-shielding Calculation. 1. A Slowing-down Resonant Model is Invoked to Determine the Absorption Reaction Rates $\tau_{a, x}^{g *}$, 2. The Background Cross Section $\sigma_{b, x}^{g}$ is Determined so that $\tau_{a, x}^{g *}=\left(\tau_{a, x}^{g *}\right)_{h}$, where the $\left(\tau_{a, x}^{g *}\right)_{h}$ are the Reaction rates Obtained with the Same Resonant Model in an Equivalent Infinite and Homogeneous Medium with

Background Cross Section $\sigma_{b, x}^{g}, 3$. The $\sigma_{b, x}^{g}$ is Used to Compute on the Fly or to Retrieve from Tabulated Values "Exact" Reaction

Rates $\tau_{r, x}^{g}$ in the Infinite and Homogeneous Medium with

Background Cross Section $\sigma_{b, x}^{g}, 4$. Multigroup Self-shielded Cross

Sections $\sigma_{r, x}^{g}$ are Determined so that a Multigroup Calculation

with these Cross Sections Reproduces the Reactions Rates $\tau_{r, x}^{g}$ represented by a homogeneous zone. This homogenized zone is constructed using the shape flux calculated on the pin cell lattice, which is also part of the needed shape flux set. The initial pivot flux can then be calculated at the assembly level with homogenized cells. The reconstructed flux is then used again to collapse the cross sections for another calculation, this time using the MOC with a detailed spatial representation. The latter is then used as pivot for the final flux reconstruction. An example of such a procedure is given in Section 10.2 where the specific flux-reconstruction operator used by AREVA is described.

A number of procedures describing 'compound operators' are available for use by APOLLO2's general users. However, the advanced user can create his/her own compound operators to better suit his/her applications. It is also possible for a user to program additional modules that are automatically integrated to produce a tailored version of the code.

\section{EXTERNAL LIBRARIES}

APOLLO2 uses external multigroup cross section libraries in its own format (APOLIB). These libraries contain temperature-dependent infinite-dilution cross sections and associated self-shielding data, fission yields, decay constants and delayed neutron data. A separate project GALILEE, [26] developed under the same roof as APOLLO2, deals with the nuclear data and provides the libraries for the code. The main processing tool comprises two standard codes, NJOY, [50] for the generation of point wise and multigroup cross sections, and CALENDF [51] for the generation of probability tables. CALENDF has been developed at the CEA and is available at the OECD/ NEA Data Bank. A dedicated utility program (N2A2), translates the NJOY and CALENDF outputs into APOLIB format. The data may come from any evaluated nuclear data file in ENDF format such as JEF, JEFF, ENDF/B and JENDL. The three standard APOLIB multigroup libraries have 99, 172 and 281 groups, respectively. The first corresponds to the energy mesh of the predecessor APOLLO code, $[27,28]$ the second is the standard XMAS structure [14] and the third is the recently optimized SHEM energy mesh. [15] New optimized multigroup meshes with increased number of groups are being realized using recent developments. [52] The code has also gamma source and gamma flux calculation capability using a 94-group gamma library with 99,172 or 281 group neutron to 94 gamma production cross sections associated to the neutron libraries.

All libraries are generated by GALILEE in full consistency with the point wise libraries used for the Monte Carlo reference code TRIPOLI-4. [24] APOLLO2 multigroup library releases have continuous-energy analogues for use by TRIPOLI-4. The standard distribution of the code comprises the neutron cross sections library based mainly on JEFF 3.1, [13] including gamma production in the 
three standard multigroup libraries and the gamma library. The more recent library contains 427 isotopes with 229 isotopes having self-shielding data.

Different library versions allow for highly accurate description of isotopic chains for the depletion calculations with energy-dependent fission yields. The latest version of the library contains 127 fission products without making use of pseudo products, contrarily to the previous major release which comprised 77 explicit and 8 pseudo products. The isotopic depletion chains can take into account the detailed transmutation related to Cobalt, Dysprosium, Erbium, Europium, Gadolinium, Iodine, Oxygen, Rhenium, Thorium and Tritium, among others. A version of a library which can be used as reference includes 310 fission products, which in turn has been validated against calculations with 683 fission products.

\section{CROSS SECTION SELF-SHIELDING METHODS}

Self-shielded cross-sections are calculated in order to preserve space-dependent multigroup reaction rates. The latter are obtained by solving a simplified elastic slowingdown equation, called heterogeneous fine structure equation, [32] written for one resonant isotope diluted in non resonant ones. Space-dependent interference effects are directly incorporated via the use of a full collision probability description with no need for geometricallydependent Dancoff factors.

The self-shielding process relies on a single-isotope external library of reaction rates tabulated versus background cross sections and temperatures. These reaction rates are computed with the NJOY Nuclear Data Processing System [50] and quadrature formulas are derived from probability tables for the calculation multigroup mesh. All these quantities are obtained from fine-multigroup calculations and are stored in the external library. The cross section self-shielding algorithm is carried out for each self-shielding region and each resonant energy group according to the following steps:

1. Because the exact resonant slowing-down operator cannot be treated in the frame of the self-shielding calculation, resonant slowing-down models allowing for a simplified solution for the fine structure flux are introduced. An approximate fine structure flux is then computed leading, thanks to quadrature formulas, to approximate partial reaction rates.

2. To correct this approximation, an equivalent homogeneous medium is found, equivalent meaning "same absorption rate when performed with the same slowing-down model." This equivalent medium is characterized by an equivalent background crosssection.

3. The "exact" heterogeneous partial reaction rates are obtained by interpolation from the reaction-rate tabulations at the equivalent background cross-section.
4. Finally, a multigroup equivalence preserving the "exact" reaction rates obtained by the homogeneous/ heterogeneous equivalence previously described gives the self-shielded cross-sections.

This procedure is applied to a heterogeneous geometry which is treated with the collision probability method. A simplification is introduced that allows to group regions in the geometry and to compute a single averaged flux for each of these groups. Every resonant isotope in one of these groups of regions defines a new self-shielded isotope in the microscopic library. [37] The flow chart in Fig. 2 illustrates a typical self-shielding calculation.

The new main improvements carried out for the modeling of cross section self-shielding in the APOLLO2 code [33] are:

- capability to self-shield resonant mixtures, taking into account the resonance overlapping between the various resonant components,

- capability to self-shield resonant scatter isotopes.

\subsection{Resonant Mixture}

To self-shield a resonant mixture, the basic idea is to consider it as a unique resonant entity and to apply the previous method. $[53,54]$ The problem is that no data (effective homogeneous tabulations or quadrature formulas) can be processed in the APOLLO2 library because the data are problem dependent. Consequently, these data must be calculated on the fly during the self-shielding process. This new treatment improves on the previous one which was iterative, each resonant mixture component being selfshielded separately.

\subsubsection{Homogeneous Tabulations}

For a resonant mixture with $M$ resonant components, with isotopic concentrations $a_{m}$, total resonant cross-sections $\sigma_{m}$ and background cross section $\sigma_{b}$, the fine structure equation reads:

$$
\left[\sum_{m=1}^{M} a_{m} \sigma_{m}(u)+\sigma_{b}\right] \varphi(u)=\sum_{m=1}^{M} a_{m} r_{m} \varphi(u)+\sigma_{b},
$$

where $\varphi(u)$ is the fine structure flux versus lethargy and $r_{m} \varphi(u)$ is the elastic resonant slowing-down operator for isotope $m$.

The "exact" solution of Eq. (1) is obtained using a fine-multigroup energy mesh $\left\{g_{f}\right\}$ for each coarse group used in APOLLO2 and by modeling the elastic resonant slowing-down operator of each isotope with the TR model [55] applied to the fine multigroup mesh. The TR approximation models elastic resonant slowing-down operators, regardless of the resonance shapes, and provides an approximation which is quasi-exact when the multigroup mesh $\left\{g_{f}\right\}$ is fine enough (about 10000 groups). With 
these assumptions:

$$
\left(\forall u \in g_{f}\right) r_{m} \varphi(u) \approx \sum_{g_{f}^{\prime} \leq g_{f}} p_{m}\left(g_{f}^{\prime}, g_{f}\right)\left\langle\sigma_{s, m} \varphi\right\rangle^{g_{f}^{\prime}}
$$

where $p_{m}\left(g_{f}^{\prime}, g_{f}\right)$ is the average probability for a neutron interacting with isotope $m$ to scatter from group $g_{f}^{\prime}$ into group $g_{f}$ and $\left\langle\sigma_{s, m} \varphi\right\rangle^{g_{s}^{\prime}}$ is the mean elastic scattering reaction rate in group $g_{f}^{\prime}$ for the isotope.

Once the flux is known, partial reaction rates for each component of the mixture are calculated on the fine mesh, via the quadrature formulas described below, and then reaction rates are collapsed on the APOLLO2 coarse mesh.

\subsubsection{Quadrature Formulas}

Quadrature formulas to calculate the partial reaction rates for each component of a mixture are needed not only for the fine multigroup mesh, as explained before, but also to determine the equivalent homogeneous medium in the multi-group mesh.

Quadrature formulas are first calculated on the fine multigroup mesh $\left\{g_{f}\right\}$ with the help of the probability tables of each component described on that mesh and making the assumption that the cross-sections of the various components are uncorrelated. The method that is used is derived from the one of CALENDF-2005. [51] These quadrature formulas are then collapsed on the coarse mesh.

\subsubsection{Remark}

Thanks to the developments carried out to treat resonant mixtures, APOLLO2 is now able to self-shield any resonant isotope without the need of effective reaction rate tabulations stored in the external library. The only data needed is the isotope probability tables described on a fine multigroup mesh.

\subsection{Scatter Isotopes}

Customarily, the elastic self-shielded transfer matrix is calculated as the product of the elastic self-shielded cross-section and the infinite dilution elastic probability transfer matrix. This approximation can lead to incorrect results for scatters such as sodium in fast reactors. [56]

In order to self-shield the transfer matrix for scatters, we recur to the solution of Eq. (1), written for one isotope (resonant mixture with one component), and directly calculate the elastic transfer matrix from group $g^{\prime}$ to group $g$ at dilution $\sigma_{b}$ with the formula:

$$
\sigma_{s}^{g^{\prime} \rightarrow g}\left(\sigma_{b}\right)=\frac{\sum_{g_{f}^{\prime} \in g^{\prime}} \sum_{g_{f} \in g} p\left(g_{f}^{\prime}, g_{f}\right)<\sigma_{s} \varphi_{\sigma_{b}}>^{g_{f}^{\prime}} \Delta u^{g_{f}}}{\sum_{g_{f}^{\prime} \in g^{\prime}}<\varphi_{\sigma_{b}}>^{g_{f}^{\prime}} \Delta u^{g_{f}^{\prime}}} .
$$

The notation here is the same as in Eq. (1) and $\left\langle\sigma_{s} \varphi_{\sigma_{b}}\right\rangle_{f}^{g_{t}^{\prime}}$, $\left\langle\varphi_{\sigma_{b}}\right\rangle^{g_{t}^{\prime}}$ and $\Delta u^{g_{t}^{\prime}}$ stand for the mean elastic scattering reaction rate, the mean flux for background cross section $\sigma_{b}$ and the lethargy interval for the group $g_{f}^{\prime}$, respectively.

Another improvement comes from the fact that for the calculation of the self-shielded elastic scattering crosssections and the self-shielded elastic transfer matrix the homogeneous/heterogeneous equivalence can be carried out for the elastic scattering reaction rates (instead of the absorption reaction rates).

\subsection{Example Calculations}

For the two validation cases described here, the reference calculations have been obtained with the Monte Carlo code TRIPOLI-4 [24] developed at CEA.

\subsubsection{Resonant Mixture}

The validation case is a MOX cell calculation, without leakage and with a plutonium content of $5.30 \%$. The effective multiplication factor and the uranium 238 and plutonium 239 absorption rates in the fuel are compared to reference results given by TRIPOLI- 4 with 6 million neutron histories. Two calculations were carried out with APOLLO2, the first one with an iterative treatment to model resonance overlapping and the second one with the mixture treatment applied from $304.32 \mathrm{eV}$ to $5.346 \mathrm{eV}$, i.e. for groups 63 to 89 in the 172-group XMAS mesh.

As far as the effective multiplication factor is concerned, the two APOLLO2 calculation results are not significantly different. The TRIPOLI-4 value is $1.17866 \pm 60 \mathrm{pcm}$, while the effective multiplication factor discrepancies are $-56 \pm 60$ and $-73 \pm 60$ for the iterative treatment and the mixture treatment, respectively.

The results concerning the reaction rates, given in Fig. 3, are much more interesting.
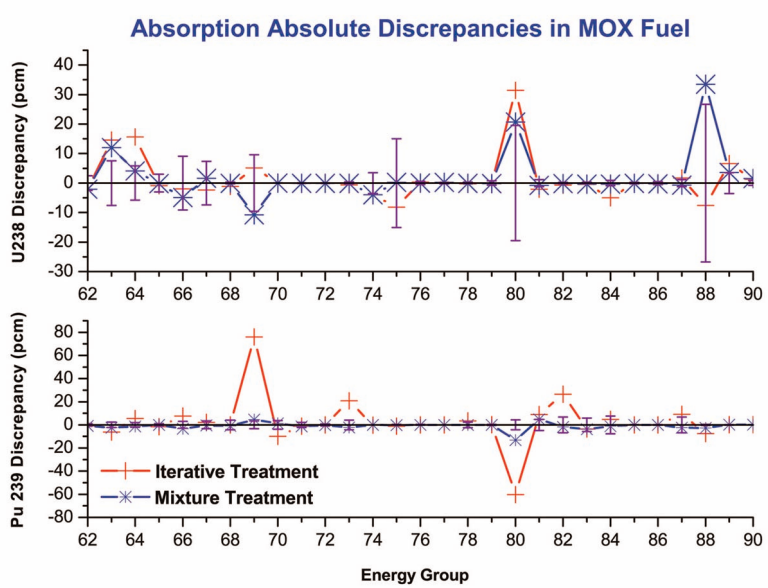

Fig. 3. MOX fuel. Absolute Discrepancies in U238 and Pu239 Absorption Rates Versus TRIPOLI-4 


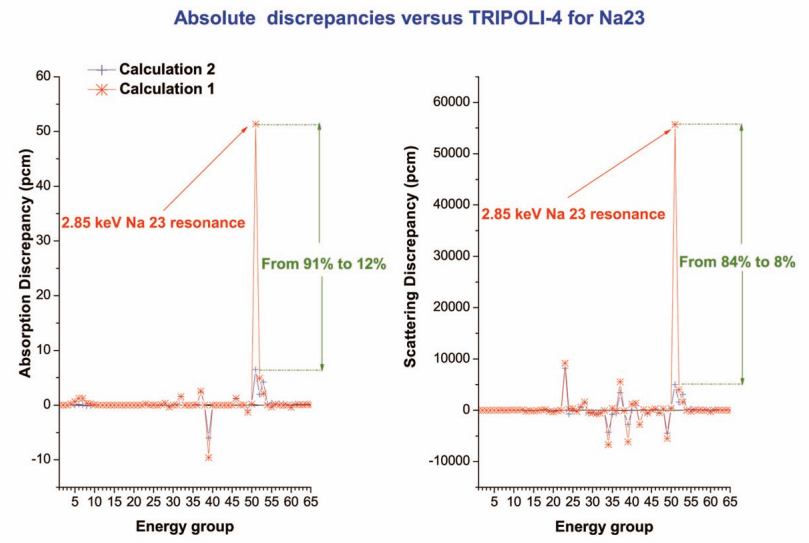

Fig. 4. Absolute Discrepancies in Na23 Reaction Rates Versus TRIPOLI-4

Because uranium 238 is the main isotope in MOX fuel, it is well calculated by the iterative method and the mixture treatment does not bring significant improvements. Contrarily, with the mixture treatment the discrepancies for the other resonant isotopes are largely reduced. For example, for plutonium 239 in groups 69 ( $67.9 \mathrm{eV}$ to $55.6 \mathrm{eV})$ and $80(22.6 \mathrm{eV}$ to $19.45 \mathrm{eV})$ the absorption discrepancies are respectively reduced from $16.44 \%$ to $0.96 \%$ and from $21.26 \%$ to $4.64 \%$, respectively.

\subsubsection{Scatter Isotopes}

The test case is an infinite lattice of pin cells featuring a fast reactor. The fuel contains uranium 238, plutonium 239 , plutonium 240 and oxygen 16 . The cladding is made of iron 56 and the coolant of sodium 23 with the homogenized wrapper tube of iron 56. [57] The reference calculation was carried out with TRIPOLI-4 using 18 million histories. Two APOLLO2 calculations have been performed with the 172-group XMAS mesh. The first calculation is a standard APOLLO2 calculation where only the scattering cross-sections are self-shielded. In the second calculation the transfer matrices are directly selfshielded.

The TRIPOLI-4 effective multiplication coefficient is $1.32151 \pm 28 \mathrm{pcm}$. For the first APOLLO2 calculation there is a discrepancy of $635 \pm 28 \mathrm{pcm}$ which is reduced to $105 \pm 28 \mathrm{pcm}$ for the second calculation.

For the reaction rates we also notice an important improvement, essentially for sodium 23, as shown in Fig. 4.

\section{FLUX RECONSTRUCTION FOR ASSEMBLY DEPLETION CALCULATIONS}

Isotopic depletion for an assembly is computed in a series of burnup or depletion steps. At each burnup step a new space-and-energy detailed assembly flux $\phi(\mathrm{r}, E)$ is computed to provide the average reaction rates for each depletion region in the assembly. [58] We shall denote this flux by $\left(\phi_{i}^{g}\right)_{B U}$, where $g$ is a fine group and $i$ is a depletion region.

We work with three transport calculations, 1D cell or simplified 2D, 2D cell-homogenized assembly and 2D heterogeneous assembly, with decreasing refinement in the energy variable. To save computation time, self-shielded cross sections and fine-group transport calculations are done for a few one-dimensional representative types of cells. To this end, cells in the assembly with a similar neutronic environment are grouped into types. Each cell type represents one or more physical cells in the assembly and, therefore, the materials for a cell type are obtained by volume averaging of the daughter physical cells. For the burnup calculation, isotopic concentrations are defined for each cell in the assembly and, within each cell, over depletion regions that we usually take to be concentric annuli.

We adopt the following notation for the energyintegrated, space-averaged fluxes obtained from a transport calculation:

- $\phi_{i}^{g}=$ flux in ring $i$ from the fine-group transport calculation for a cell of a given type,

- $\phi_{I}^{G}=$ flux in cell $I$ from the intermediary coarse-group 2D cell-homogenized assembly transport calculation,

- $\phi_{r}^{q}=$ flux in region $r$ from the large-macrogroup 2D heterogeneous assembly calculation.

The depletion flux $\left(\phi_{i}^{g}\right)_{B U}$ is reconstructed by combining the fine-group fluxes $\phi_{i}^{g}$, which are the most detailed energy representation of the pin cells spectra, with the heterogeneous-assembly large-macrogroup flux $\phi_{i}^{c}$. The correction is done to account for the specific position of each daughter cell in the assembly. We have explored several possibilities but the best results were obtained from

$$
\left(\phi_{i}^{g}\right)_{B U}=\phi_{i}^{\mathcal{G}} \times\left(f_{i}^{g}\right)_{w}, g \in \mathcal{G},
$$

where the fine-group shape factors

$$
\left(f_{i}^{g}\right)_{w}=\frac{\left(\phi_{i}^{g}\right)_{w}}{\sum_{g \in \mathcal{G}}\left(\phi_{i}^{g}\right)_{w}}, g \in \mathcal{G}
$$

are used to collapse the cross sections for the 2D heterogeneous-cell assembly flux calculation: $\Sigma_{i}^{\mathcal{G}}$ $=\sum_{g \in \mathcal{G}} \Sigma_{i}^{g}\left(f_{i}^{g}\right)_{w}$. Hence, the reconstructed flux $\left(\phi_{i}^{g}\right)_{B U}$ not only preserves the fine-spatial distribution of the pivot flux $\phi_{i}^{\mathcal{G}}$ but it also conserves the coarse-group reaction rates

$$
\sum_{g \in \mathcal{G}} \Sigma_{i}^{g}\left(\phi_{i}^{g}\right)_{B U}=\Sigma_{i}^{\mathcal{G}} \phi_{i}^{\mathcal{G}} .
$$


In order to account for environment effects, an intermediary macrogroup 2D cell-homogenized calculation is used to improve the spatial resolution of the fine-group flux. Instead of using the flux $\left(\phi_{i}^{g}\right)_{w}=\phi_{i}^{g}$ in Eq. (4) we use the reconstructed flux

$$
\left(\phi_{i}^{g}\right)_{w}=\phi_{I}^{G} \times f_{i}^{g}, g \in G, i \in I,
$$

where

$$
f_{i}^{g}=\frac{\phi_{i}^{g}}{\sum_{g \in G} \phi_{i}^{g}}, g \in G
$$

The cross sections for the intermediary calculation are obtained by collapsing of the fine-group fluxes, $\Sigma_{i}^{G}$ $=\sum_{g} \in G \sum_{i}^{g} f_{i}^{g}$.

\subsection{Leakage Corrections}

Leakage corrections have to be introduced to account for the macroscopic variation of the flux in the critical core. In flux reconstruction procedures leakage can be introduced at one or more stages of the calculation. We have found that the best results are obtained by introducing a leakage correction in the reconstructed fine flux used to collapse the cross sections and also in the final burnup flux. We use the flux (5) to homogenize the assembly and compute the infinite medium spectrum $\phi_{\infty}^{g}$ and the associated critical leakage cross section $D^{g} B^{2}$. Next, the flux $\left(\phi_{i}^{g}\right)_{w}$ is used to compute the spatial shape factors

$$
\widehat{f}_{i}^{g}=\frac{\left(\phi_{i}^{g}\right)_{w}}{\sum_{i}\left(\phi_{i}^{g}\right)_{w} V_{i}}
$$

where the sum is over all regions in the assembly and $V_{i}$ is the volume of region $i$. These factors are then used to normalize $\left(\phi_{i}^{g}\right)_{w}$ as follows

$$
\left(\phi_{i}^{g}\right)_{w} \rightarrow \phi_{\infty}^{g} V \times \widehat{f}_{i}^{g}
$$

with $V=\sum_{i} V_{i}$.

It is this scaled flux that is used for cross section collapsing and to define the energy shape factors in Eq. (4) letting to the burnup flux $\left(\phi_{i}^{g}\right)_{B U}$. The $D B^{2}$ cross section is also collapsed and incorporated in the heterogeneous assembly calculation. Finally, the reconstructed burnup flux is similarly corrected with its infinite medium critical spectrum $\left(\phi_{\infty}^{g}\right)_{B U}$ :

$$
\left(\phi_{i}^{g}\right)_{B U} \rightarrow\left(\phi_{\infty}^{g}\right)_{B U} V \times \frac{\left(\phi_{i}^{g}\right)_{B U}}{\sum_{i}\left(\phi_{i}^{g}\right)_{B U} V_{i}}
$$

\subsection{Example Calculations}

We have analyzed a BWR MOX depletion benchmark for $0 \%, 40 \%$ and $70 \%$ void fraction. [59] The geometry of this benchmark, depicted in Fig. 5, is that of a $10 \times$ 10 unrodded assembly with an internal Atrium-like water channel and diagonal symmetry. The assembly contains 77 MOX pins with 5 different enrichments and 14 Gadolinia bearing rods. Because of the diagonal symmetry only 49 pins have to be considered for the burnup calculations.

\subsubsection{Flux Reconstruction Calculation}

To account for the different neutronic environments in the assembly, the central zone around the water channel and the peripheral zone, we have defined 10 types of cells (8 MOX and 2 Gadolinia). After each burnup step these 10 cells are constructed by volume averaging of the daughter cells. The fine-group calculation was done with the 172-group ( 80 thermal) XMAS library with a fast interface-current 'multicell' $\mathrm{UP}_{1}$ flux solver where cell exchanges account for the different positions of the daughter cells in the assembly. [32] The auxiliary cellhomogenized assembly calculation was done with the IDT short-characteristics module of APOLLO2 with 40 macrogroups ( 25 thermal) and $16 \times 16$ cells, while the 16-macrogroup (13 thermal) detailed heterogeneousassembly calculation was done with the method of (long) characteristics (MOC) of the TDT module with 3784 regions. [48],[4]

Self-shielded cross sections accounting for isotopic mixture and spatial interactions are computed in $1 \mathrm{D}$

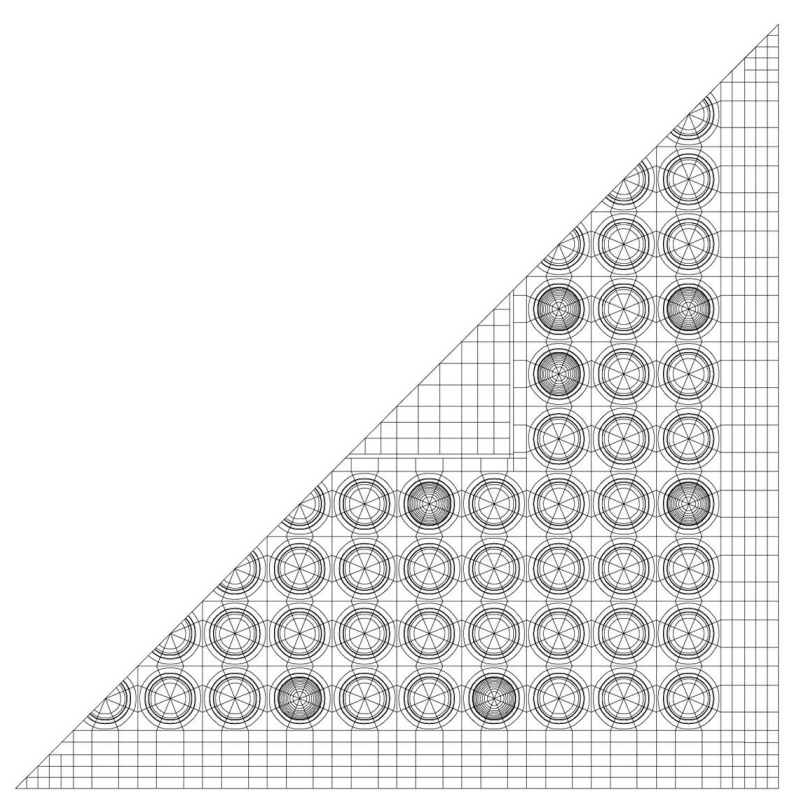

Fig. 5. Geometry of the BWR MOX Benchmark Showing the Partition in Regions for the MOC Calculation 
cylindrical geometry for each cell type. [53] Then, improved self-shielded cross sections for the U238 are obtained from a multicell $\mathrm{UP}_{1}$ calculation with the 10 cell types. Finally, for the multicell calculations, the water channel box and surrounding water was replaced by flux averaging of the water-box-water region of a 1D slab (water-box-water-assembly fuel) calculation with an assembly averaged fuel obtained from a 1D cylindrical calculation for a volume-averaged cell. Self-shielded cross sections for the water box were obtained from a 1D slab (water-box-water) calculation.

\subsubsection{Validation of the Reference Calculation}

The reference calculation for the depletion run was done with the XMAS library using the TDT solver with the same number of regions and with the same self-shielded cross sections as for the flux reconstruction calculation. We validated this calculation by a comparison with the Monte Carlo TRIPOLI-4 code for the startup assembly. [24] The results shown in Table 1 and Fig. 6 confirm the accuracy of the reference calculation as well as that of the flux reconstruction scheme. The RMS values in Table 1 are defined as

$$
R M S=\sqrt{\frac{\sum_{i=1}^{N}\left(1-\frac{c a l}{r e f}\right)^{2}}{N} .} .
$$

Table 1. Reference and Reconstructed Results Compared to TRIPOLI-4 for the BOL BWR Assembly. Pin Reaction Rates Error Bounds and RMS in \%

\begin{tabular}{|c|c|c|c|}
\hline & $\begin{array}{c}\text { fission rates } \\
\text { (min,max) RMS }\end{array}$ & $\begin{array}{l}\text { absorption rates } \\
(\min , \max ) \mathrm{RMS}\end{array}$ & $\Delta k_{\text {eff }}(\mathrm{pcm})$ \\
\hline reference & $(-0.7,1.4)$ & $(-0.9,1.0)$ & -87 \\
\hline reconstructed & $(-1.1,1.7)$ & $(-1.0,1.6)$ & 163 \\
\hline
\end{tabular}

\subsubsection{Depletion Calculations}

We have run depletion calculations up to a burnup of $70 \mathrm{GWd} / \mathrm{t}$ for the three void fractions of 0,40 and $70 \%$. Each calculation comprised 3 initial steps with small burnups increments, to account for fast Gadolinia burnup, and 85 steps every $250 \mathrm{MWd} / \mathrm{t}$. Because our depletion calculation uses a predictor-corrector technique, 2 of the 88 steps were recalculated given a total of 90 flux calculations. The results obtained with the reconstruction technique were compared to those from the reference calculation. In a DEC-ALPHA at $1150 \mathrm{MHz}$ the running time for an entire depletion calculation was $1 \mathrm{~h} 21 \mathrm{~min}$ for the reconstruction technique as compared to $12 \mathrm{~h} 30 \mathrm{~min}$ for the reference calculation. A summary of the comparisons is shown in Fig. 7. The errors in reactivity versus burnup (left graphic) are dominated by the overestimation of Gadolinia burnup. The reactivity error during the burnup calculation does not exceed $90 \mathrm{pcm}$ and the maximum error in pin powers does not exceed 1\%. The RMS for the pin power distribution was smaller than $0.5 \%$.

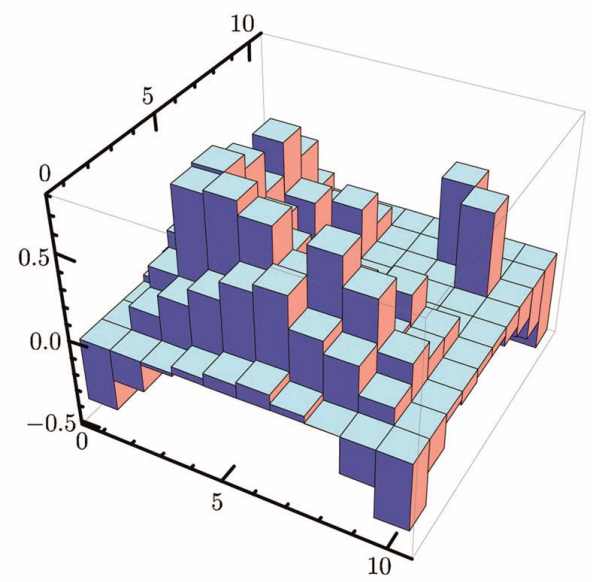

Fig. 6. BOL BWR Assembly. Reconstructed Absorption Errors (pcm) with Respect to the Reference Solution

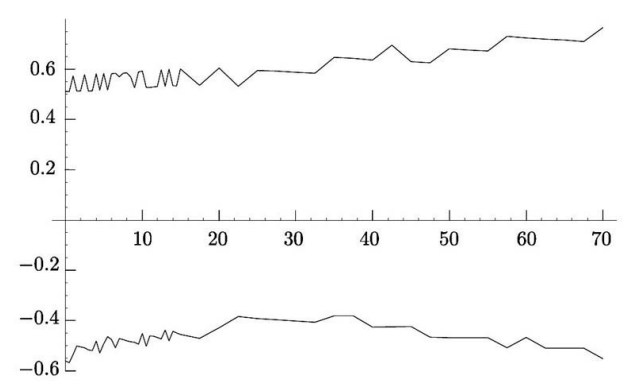

Fig. 7. Comparison of Reconstructed and Reference Depletion Calculations. Left: Reactivity Errors (pcm) Versus Burnup (MWd/t) for 0, 40 and 70\% void Fractions. Right: Fission Rate Error Bounds (\%) Versus Burnup (MWd/t) for 40\% Void Fraction 


\section{COLLISION PROBABILITY METHOD}

A new development has been done for the calculation of the RZ geometry with the collision probability method (CPM). [60] We consider one-group transport with isotropic scattering in a heterogeneous domain $D$ with boundary $\Gamma$. The domain is partitioned into a set of homogeneous regions that define on its boundary $\Gamma$ a set of surfaces. We assume that the external sources are isotropic and uniform in each region and that neutrons enter the domain isotropically and uniformly through each surface. The formulation of the flat-flux collision probability method for general 3D geometries can be written as:

$$
V_{i} \Phi_{i}=\sum_{j} P_{i j} F_{j}+\sum_{\alpha} I_{i \alpha} J_{\alpha}^{-}
$$

where the sums in $j$ and $\alpha$ are, respectively, over all the regions in the domain and over all the surfaces on its boundary and

- $V_{i}=$ volume or homogeneous region $i$,

- $\Phi_{i}=$ average flux in region $i$,

- $P_{i j}=$ generalized collision probability from region $j$ into region $i$,

- $F_{i}=\Sigma_{s i} \Phi_{i}+S_{i}=$ average emission density in region $i$,

- $I_{i \alpha}=$ generalized 'incoming' probability from surface $\alpha$ into region $i$,

- $J_{\alpha}^{-}=$current entering surface $\alpha$.

Matrix coefficients $P_{i j}$ and $I_{i \alpha}$ are computed via a numerical integration over the area $S_{\perp}(\Omega)$ transverse to the neutron trajectory:

$$
P_{i j}=K f_{i j}, I_{i \alpha}=\left(4 / A_{\alpha}\right) K f_{i \alpha},
$$

where $K$ indicates the integration over the transverse plane and the $f_{i j}$ and $f_{i \alpha}$ are analytical expressions to be evaluated over each of the numerical trajectories. For example, with $\tau_{i}$ the optical thickness across region $i$,

$$
f_{i j}=\left\{\begin{array}{cc}
\frac{1-e^{-\tau_{i}}}{\Sigma_{i}} e^{-\tau_{i j} \frac{1-e^{-\tau_{j}}}{\Sigma_{j}}}, & i \neq j, \\
2 \frac{\tau_{i}-\left(1-e^{-\tau_{i}}\right)}{\Sigma_{i}^{2}}, & i=j .
\end{array}\right.
$$

In 3D Cartesian coordinates, every trajectory is contained in a vertical plane. Therefore, the trajectory can be defined by the coordinates $(\rho, \varphi)$ of the polar vector $\rho$ orthogonal to the plane and by its local plane coordinates $(z, \theta)$. In these coordinates $d \Omega=d \varphi \sin \theta d \theta$ and $d S_{\perp}=$ $d \rho d z_{\perp}$ with $z_{\perp}=z \sin \theta$. For RZ geometries the integration over $\varphi$ gives a factor $\pi$ and we have

$$
K_{R Z} f=\frac{1}{2} \int_{0}^{R} d \rho \int_{0}^{\pi} d \theta \sin \theta \int d z_{\perp} f\left(\rho, \theta, z_{\perp}\right) \sim \frac{1}{2} \sum_{t} w_{t} f(t)
$$

where the rightmost expression indicates that all the integrals are done by numerical discretization in $\rho, \theta$ and $z_{\perp}$. To generate the trajectories for each numerical ordinate $\rho$ we compute the intersection of the geometric mesh with the corresponding vertical plane. As shown in Fig. 8 this reduces the problem to a series of twodimensional trackings over a Cartesian mesh.

The main difficulty is the correct treatment of axial boundary conditions. In this work we have introduced compound trajectories. [4] This tracking technique ensures reciprocity and minimizes tracking storage and the subsequent numerical effort to compute the collision matrices. However, the tracking strategy is much more complicated that the one for single trajectories and depends on the type of geometrical motions applied as boundary conditions, in our case specular reflection and translation on the axial surfaces. The example in Fig. 9 illustrates the

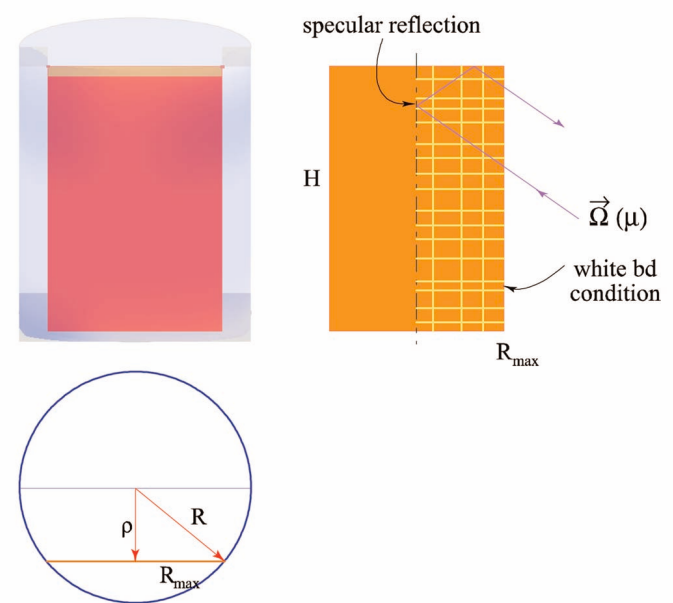

Fig. 8. A Radial Quadrature Abscissa Defines a Tangent Plane where Tracking in $\mu$ and $z$, has to be Done. The Figure Shows the Intersections of the RZ Regions with the Cut Plane.

A Trajectory is Shown for the Case of Specular Reflection on the Top Boundary

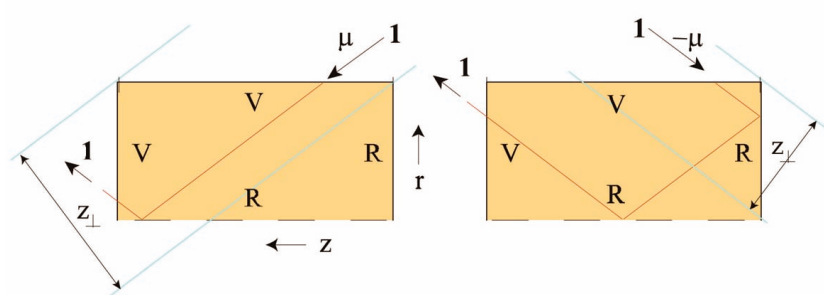

Fig. 9. RZ Geometry with Top Albedo Condition (V) and Bottom Specular Reflection (R). Trajectories Entering with $\mu$ $>0$ (Left Figure) are Only Tracked for the Domain $z_{\perp}$, While Trajectories Entering in Direction $-\mu$ (Right Figure) are Tracked for the $z_{\perp}$ Range Indicated in the Figure. One Example for Each Case is Shown as Trajectory 1 
tracking technique.

The method accounts for white albedo, specular and translation boundary condition on the top and bottom surfaces of the geometry and for a white albedo condition on the outer radial surface. It has been applied to the calculation of multigroup self-shielded cross sections for gray Gadolinia fuel pin absorbers in BWRs. [60]

\section{METHOD OF CHARACTERISTICS}

The new developments concern both the method of short and long characteristics (MOC). A new structure of heterogeneous cells was incorporated in module IDT. A cell may contain now an arbitrary number of concentric annuli subject to the condition that the most external one does not intersect the boundaries of the cell, allowing thus the explicit representation of the fuel pins. At the present time this extension has been done only for the $\mathrm{XY}$ geometry. [61] Constant, linear and bilinear $(1, x, y, x y)$ angular flux expansions can be used in the regions of the cell. For every angular direction the associated collision, escape, incoming and transmission matrices are computed by projecting all cell discontinuities on the transverse direction and by using a Gauss-Legendre quadrature on each transverse segment. Figure 10 shows a detail of the numerical integration used for the calculation of the matrices. Moreover, a non-linear acceleration technique has been implemented for the acceleration of the transport iterations. This technique, based on cell homogenization, uses a diffusion-like equation, much alike to the CMFD method, [63] but with surface Eddington factors. [64]

As an example calculation we present in Table 2 and Fig. 11 results for the 7-group NEA/OECD C5G7 MOX 2D benchmark [65] obtained by subdividing the fuel cells with 1 or 2 internal rings. The results confirm that the use of heterogeneous cells gives a good representation of the

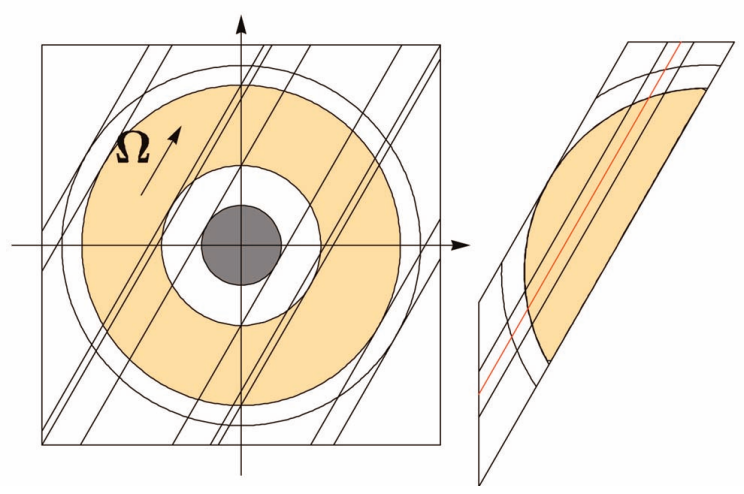

Fig. 10. Integration Scheme for the Calculation of IDT Heterogeneous-cell Matrices. Left: Projection of Cell Discontinuities on the Transverse Propagation Direction. Right: Detail of One of the Trajectories used in a Cell Slide flux in the pin cells, especially with the linear and bilinear expansions. For the 1-ring calculation, the bilinear expansion yields a maximum error on the fission rate lesser than $2 \%$, demonstrating the good accuracy of the method.

An improved $\mathrm{DP}_{1}$ synthetic acceleration technique has been developed for use with the method of long characteristics. [66] This technique extends the previous $\mathrm{DP}_{0}$ method by introducing a double $\mathrm{P}_{1}$ angular expansion for the angular fluxes entering and exiting the regions. A biconjugate gradient stabilized Krylov method [67] is used, together with algebraic pre-conditioners, [68] for the iterative solution of the $\mathrm{DP}_{N}$ equations. Further speed up of the solution of the $\mathrm{DP}_{N}$ equations has been obtained via a multi grid surface-response method based on a multidomain decomposition. [69] Furthermore, the synthetic acceleration is now used for the initialization and the acceleration of multigroup thermal and external iterations. [69]

Finally, a new spatial discretization technique has also been implemented in the method of long characteristics.

Table 2. C5G7 MOX Benchmark Results Compared to the Monte Carlo Reference. NR: Number of Regions; Order: Linear (L), Bilinear (BL); CPU: Computing Time in a Dual-core Intel Xeon 2x2MB L2 Cache $3.80 \mathrm{GHz}$; RMS: Fission-rate Root Mean Square Error; |errl: Maximum Fission-rate Error, $\Delta \rho$ : Reactivity Error

\begin{tabular}{l|c|c|c|c|c|c}
\hline Calculation & \multirow{2}{*}{ NR } & order & $\begin{array}{c}\text { CPU } \\
(\mathrm{s})\end{array}$ & $\begin{array}{c}\text { RMS } \\
(\%)\end{array}$ & $\begin{array}{c}\mid \text { err } \\
(\%)\end{array}$ & $\begin{array}{c}\Delta \rho \\
(\mathrm{pcm})\end{array}$ \\
\hline \multirow{2}{*}{ IDT 1-ring } & \multirow{2}{*}{3757} & $\mathrm{~L}$ & 13.6 & 0.59 & 1.62 & 4 \\
\cline { 3 - 7 } & & $\mathrm{BL}$ & 13.3 & 0.41 & 1.12 & 7 \\
\hline \multirow{2}{*}{ IDT 2-ring } & \multirow{2}{*}{4913} & $\mathrm{~L}$ & 16.4 & 0.45 & 1.57 & 3 \\
\cline { 3 - 7 } & & $\mathrm{BL}$ & 17.9 & 0.32 & 0.96 & 5 \\
\hline
\end{tabular}

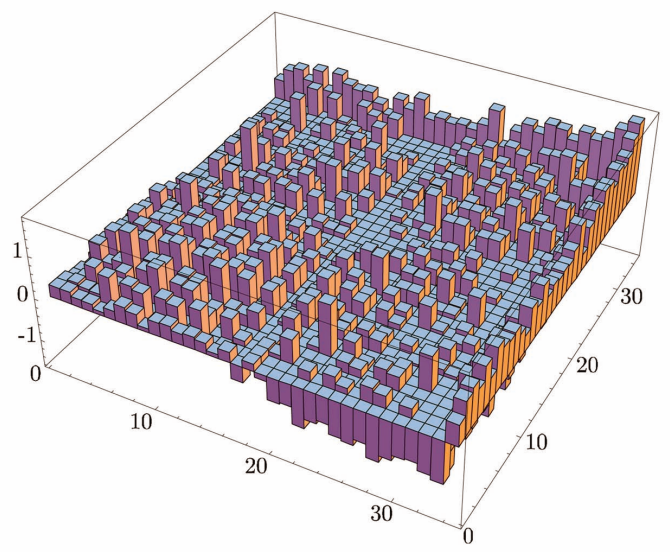

Fig. 11. Fission Rate Relative Error (\%) for the Central Bundle of the C5G7 MOX Benchmark 
Here surface angular flux moments are interpolated linearly along each trajectory to provide an improved linear surface source (LS) approximation. Because this is a trajectorydependent source approximation, a renormalization of the surface angular flux moments is necessary in order to achieve region conservation. [70] Contrarily to the usual step approximation, the LS scheme allows using larger regions and therefore a lighter tracking, leading thus to a reduction of computing time for equivalent accuracy. Typically, for infinite lattice assembly calculations the region partition comprises a region per burnup medium in each pin cell. A further improvement is achieved by calculating the weight associated with the intersection of a trajectory with a surface as the product of the weight associated with the trajectory (usually a constant for uniformly spaced parallel trajectories) times the effective proportion of the surfaces 'viewed' by the trajectory. [71] This technique was inspired from a previous analysis whose aim was to minimize tracking for whole-core MOC calculations. [72]

As an example calculation we consider two typical $17 \times 17$ PWR UOX and MOX assemblies [71]. The calculations were done with 281-group SHEM multigroup cross sections based on JEFF3.1 [73] nuclear data evaluation, with self-shielding of resonant mixtures in the energy domains $22.5 \mathrm{eV}-2.2 \mathrm{KeV}$ and $22.5 \mathrm{eV}-7.5 \mathrm{KeV}$ for the $\mathrm{UO} 2$ and MOX assemblies, respectively, and $\mathrm{P}_{1}$ scattering anisotropy. Eigenvalue lattice calculations were carried out for the two assemblies with the step (SC) and LS MOC methods and compared to reference continuousenergy TRIPOLI-4 results. All MOC calculations were done with an angular product quadrature formula with 24 uniformly spaced azimuthal angles and 2 polar BickleyNaylor angles [74] and a uniform transverse integration step of $0.1 \mathrm{~cm}$. The total numbers of regions used for the SC approximation were 801 and 1578, respectively, for the UOX and the MOX assemblies, while these numbers were reduced to 258 for the LS approximation for both assemblies. The 258-region partition was constructed by using a total of four rings to represent the four depletion media in each fuel pin cell. Figure 12 depicts the region partition used for the SC and the LS methods for the calculation of the MOX assembly with $1 / 8$ symmetry.

A further simplification consists of reducing the number of regions by volume-homogenizing two or more regions and recovering the region-averaged fluxes from the linear surface expansion obtained from the LS calculation with homogenized regions. For the UOX and MOX assemblies this approximation was used to reduce the number of rings per pin to two rings, resulting in a total of 180 regions. Finally, an improved surface representation for the angular flux moments was achieved by replacing the previous constant-surface approximation by a piecewise-constant approximation obtained by subdividing each surface of a region into several pieces. [71] The homogenization technique with the LS approximation was first tested against a reference TRIPOLI-4 calculation. We present in Fig. 13 a comparison between the 4-ring and the 2-ring LS burnup calculations for the UOX and MOX lattices up to 80000 $\mathrm{MWd} / \mathrm{t}$. The total computation times for the $\mathrm{SC}$ and for the 4- and 2-ring LS approximations were, respectively, $1 \mathrm{~h} 26 \mathrm{~m}, 40 \mathrm{~m}$ and $33 \mathrm{~m}$ for the UO2 burnup calculations
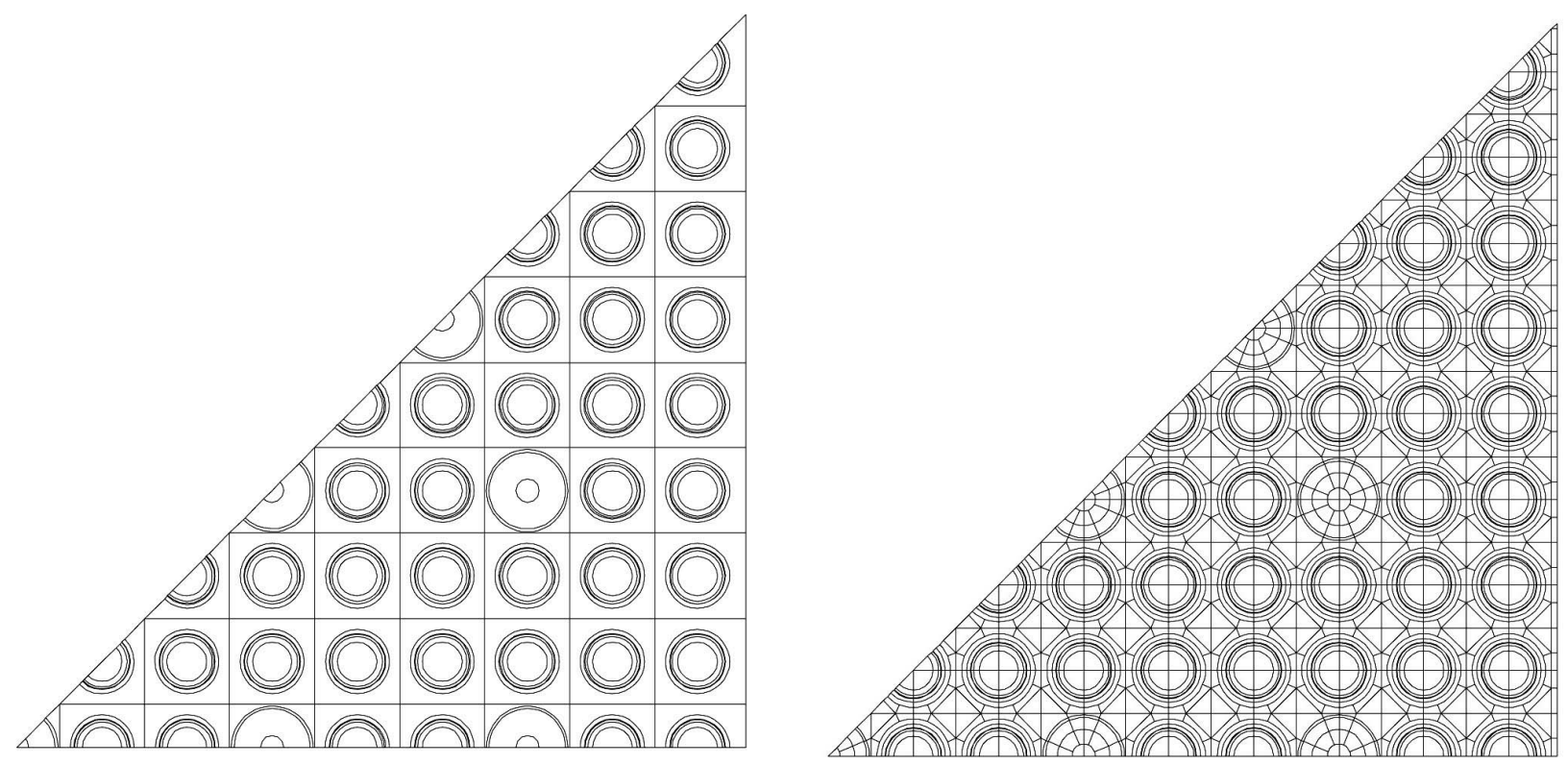

Fig. 12. Detail of Spatial Discretization for the MOX Assembly. Left: 4-ring LS Discretization with 258 Regions. Right: Step Discretization with 1578 Regions 
and $1 \mathrm{~h} 56 \mathrm{~min}$, 55min and $41 \mathrm{~min}$ for the MOX burnup calculations. The result shows that the LS approximation reduces the computational cost by a factor of two.

\subsection{Overall Method Comparison}

We run standard infinite lattice calculations for several PWR assemblies, including UOX and MOX fuel as well as voided conditions, using the collision probability method (CPM) and the methods of short and long characteristics For every type of assembly, all the calculations were done with the same multigroup cross section data based on the SHEM 281-group mesh, the same self-shielding technique and $\mathrm{P}_{1}$ scattering anisotropy (except CPM that used $\mathrm{P}_{0}$ transport corrected scattering.) Self-shielding was done using the mixture shielding formalism. For the UOX fuel, including also the gadolinium, the U235 and U238 resonance interference was treated in the range $22.5 \mathrm{eV}$ $2.22 \mathrm{keV}$, while for the MOX fuel resonance interference was done in the range $22.5 \mathrm{eV}-7.46 \mathrm{keV}$ for the isotopes U238, Pu239, Pu240 and Pu241. Reference results were obtained from continuous-energy TRIPOLI-4 calculations.

Hence, all flux solvers used the same effective multigroup cross sections. The results for the different problems are schematically compared in Fig. 14. The CPM calculations were done with an approximate interface double $\mathrm{P}_{1}$ technique and full collision matrices per cell. As expected this crude calculation was the fastest and, because of compensation effects, it yielded the best $\mathrm{k}_{\text {eff }}$ results. Indeed, for most of the assemblies considered the effect of the anisotropy on the $\mathrm{k}_{\text {eff }}$ value requires the use of a $\mathrm{P}_{5}$ order to get Monte Carlo accuracy. [71] However, this effect is less important on the detailed reaction-rate map and, in particular, on the power distribution. The results in the figure show a consistent good performance for the long characteristic methods. The results obtained with the short characteristic method (SCM) used the new heterogeneous-cell approximation which has not been
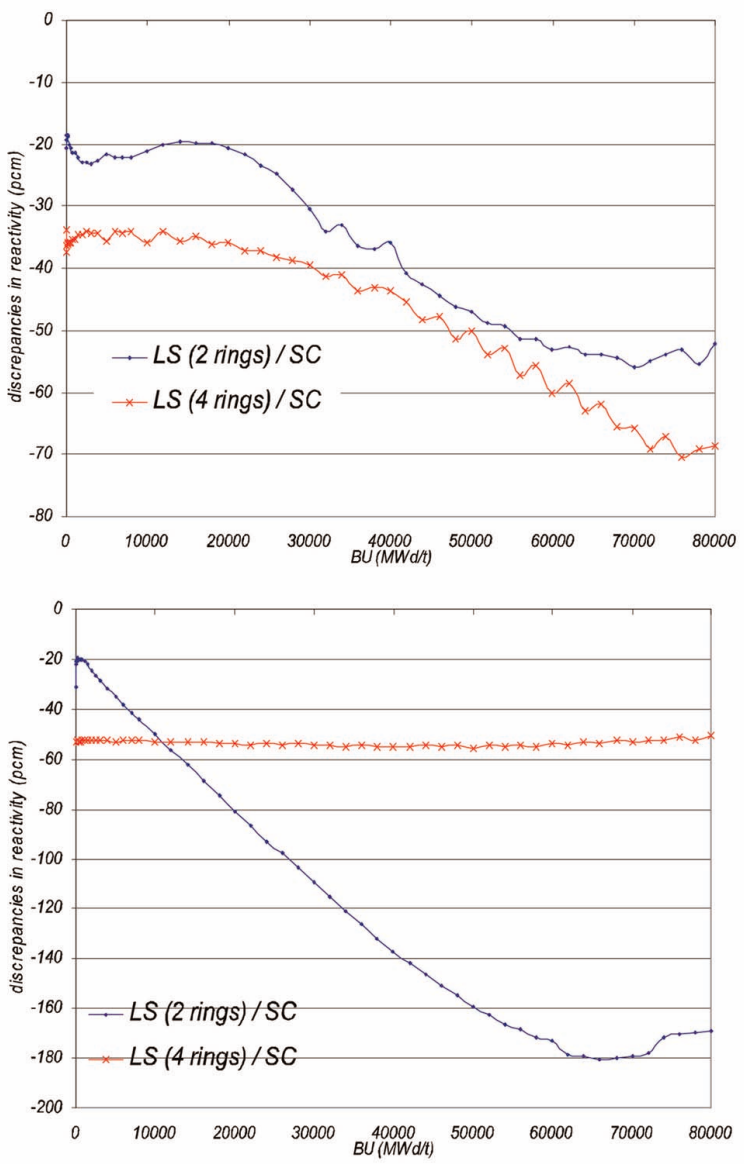

Fig. 13. Discrepencies on Reactivity Between the LS MOC Calculations using 4 Rings (Red) and the Homogenized LS MOC Calculations with 2 Rings (Blue) Per Pin. Top: UOX Lattice. Bottom: MOX Lattice

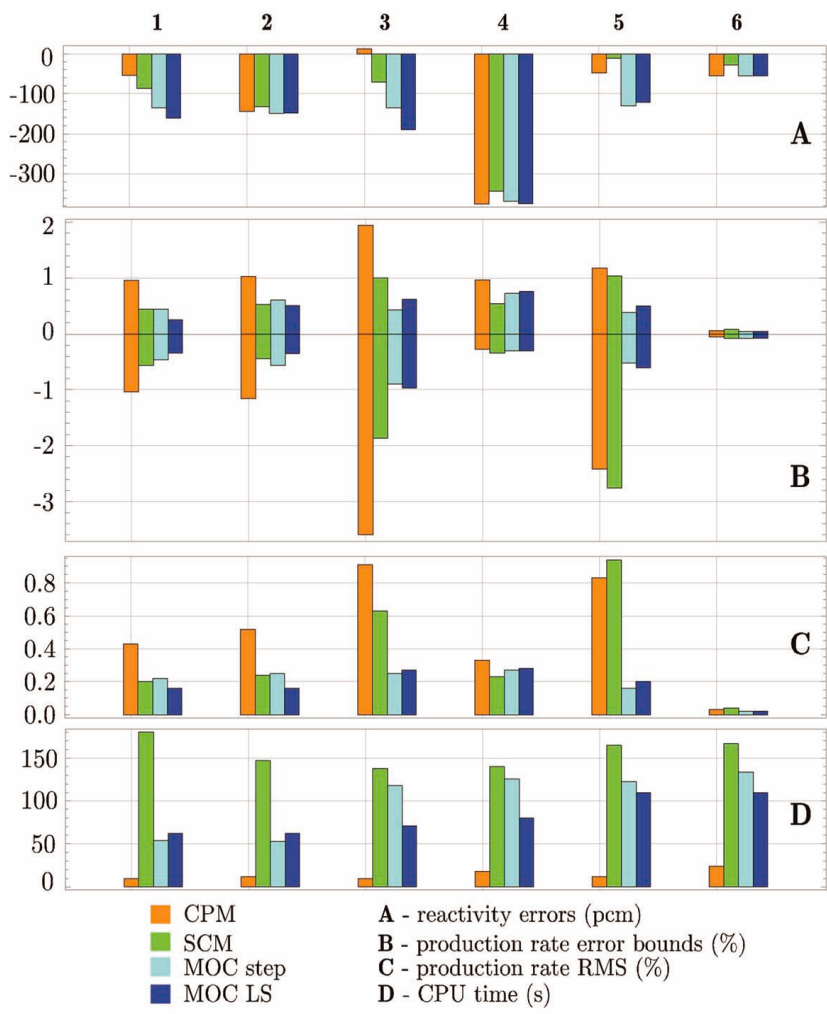

Fig. 14. Method Comparisons for PWR UOX and MOX Assemblies. Reference from a Continuous-energy TRIPOLI-4 Calculation. 1: Standard $17 \times 17$ Pin UOX Assembly; 2: $17 \times$ 17 UOX Assembly with Gadolinium Bearing Fuel Pins; 3: 17 $\times 17$ MOX Assembly with Three Zones of Different Pu Content; 4: Configuration 3 in a Voided Condition; 5: Tightly Packed MOX Assembly with $19 \times 19$ Cells and Uniform Pu Enrichment; 6: Configuration 5 in a Voided Condition 
fully optimized yet. For this method the time needed for the evaluation of the matrices rapidly increases with the number of groups and reaches near one half of the total computation time for the cases here discussed. The high calculation time was penalized because for heterogeneous cells: a) at the present time there is no acceleration for the external iterations and $b$ ) the current multigroup loop used in IDT to compute the different cell matrices groupper-group is not optimized (a calculation done for several groups at once could reduce by $25 \%$ the time needed for matrix evaluation.) The precision given by some of the SCM cases is also affected by the fact that subdivision of surfaces into smaller ones is not available in the present version.

\section{TWO-DIMENSIONAL CORE CALCULATIONS}

Core calculations with APOLLO2 with the method of characteristics have been made possible thanks to two major developments: the linear surface flux expansion and the pre conditioned multi grid surface-response method $\mathrm{DP}_{N}$ acceleration. Our first example calculation, for a 900 MWe PWR with 1/8 symmetry, is depicted in Fig. 15.

The core contains six different assemblies with varying burnup. Every pin in the assemblies was fully described and a detailed discretization was used for the reflector structures surrounding the core. First, a 281-group interface-current $\mathrm{CP}$ infinite-lattice calculation was done per each type of assembly to produce a 26-group cross section library Then, two 26-group MOC calculations, with the SC and the LS approximations, were carried out and the results were compared to continuous-energy TRIPOLI-4 reference calculations. The assembly detailed meshes used for the SC and the LS calculations are shown in Fig. 16.

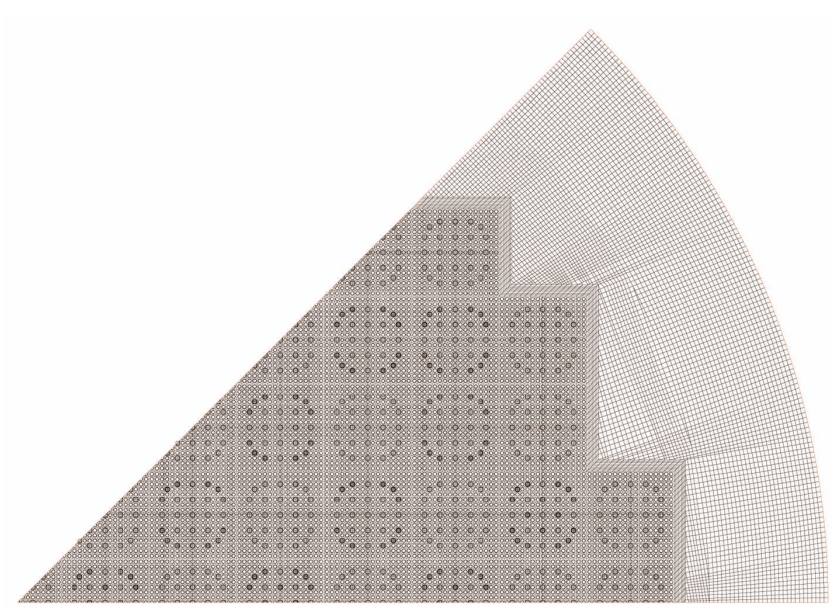

Fig. 15. Computing Mesh for the PWR (1/8 Symmetry)
The comparison was done for the $\mathrm{k}_{\text {eff }}$ and the production rates per quarter of assembly. The standard deviations for TRIPOLI-4 reference results were $5 \mathrm{pcm}$ for the $\mathrm{k}_{\text {eff }}$ and $0.1 \%$ for the production rates. A short summary of the results is given in Table 3, while a detailed production-rate map is shown in Fig. 17 for the LS calculation.
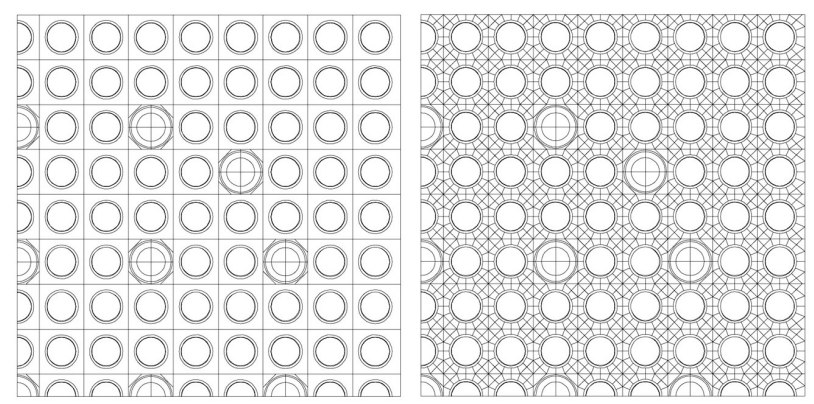

Fig. 16. Detail of Spatial Discretization for the PWR $900 \mathrm{MWe}$ Assemblies. Left: LS Discretization with 413 Regions. Right: Optimized Step Discretization with 1925 Regions

Table 3. PWR 900 MWe with 1/8 Symmetry. Step (SC) and Surface-linear (LS) MOC Results Compared to TRIPOLI/4. Quarter-assembly Production Rates Maximum Error and RMS in \%. Computing Times are for an AMD Opteron Processor 8482197 Mhz with 1024 Kb Cache

\begin{tabular}{|c|c|c|c|c|}
\hline MOC & \# regions & $\begin{array}{c}\Delta k_{e f f} \\
(\mathrm{pcm})\end{array}$ & $\begin{array}{l}\text { production rates } \\
\max \text { RMS }\end{array}$ & CPU (s) \\
\hline $\mathrm{SC}$ & 162949 & -245 & 2.6 & 6660 \\
\hline LS & 44289 & -297 & 2.9 & 3843 \\
\hline
\end{tabular}

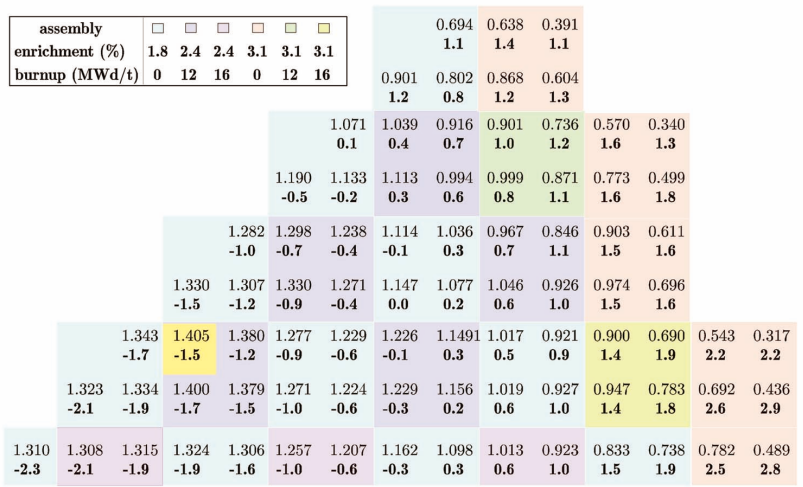

Fig. 17. PWR 900 MWe 1/8 Symmetry with Six Types of Assemblies. Comparison of LS MOC Production Rates Per

Quarter Assembly to TRIPOLI-4 Reference Values. Top: TRIPOLI-4 Values. Bottom (bold): Relative Errors (\%). Small Yellow Box: Peak Production Rate 
Our second example is the calculation of a high temperature gas-cooled prismatic reactor (HTGR). The geometry depicted in Fig. 17 for 1/12 core symmetry comprises the internal and external graphite reflectors and 9 fuel assemblies with explicit representation of the fuel compacts. A single type of kernel partitioned into seven regions was used for each compact (with three flux regions in the fuel grain), resulting in a total of 39216 regions. A MOC 99-group BOL calculation took 9 hours in a DEC alpha computer. The double-heterogeneity method, recently developed for the MOC, [43 - 46] was used for cross-section self-shielding as well as for the final multigroup calculation.

The results were compared with a reference TRIPOLI-4 calculation. The $\mathrm{k}_{\text {eff }}$ values were $1.4316( \pm 32$ $\mathrm{pcm})$ and 1.42881 for TRIPOLI- 4 and for the MOC calculations, respectively, resulting in an error of -195 $\mathrm{pcm}$ for the latter. A comparison of the normalized assembly reaction rates is given in Table 4 . Once the MOC calculation was validated at BOL, APOLLO2 was used as a reference for a depletion calculation with the diffusion code CRONOS2. [75] A more detailed 172group calculation for a $1 / 6$ symmetry and with over 40000 regions (comprising about 500 burnable fuel zones and 60 zones with the B4C control absorbers whose isotopic depletion was also followed) is given in Ref. [76].

\section{ADDITIONAL FEATURES}

In this section we summarize some of the relevant functions and tools developed for the APOLLO2 code:

- The code can calculate the reactivity break-down by comparing a pair nominal and perturbed calculations. [49] Sensitivity coefficients can be computed for a user given isotope, reaction, energy interval and spatial domain. The perturbation integrals are calculated using the discrete ordinates approximation of the angular flux with the finite-differences or the MOC solvers.

- First-order perturbation theory can be employed using

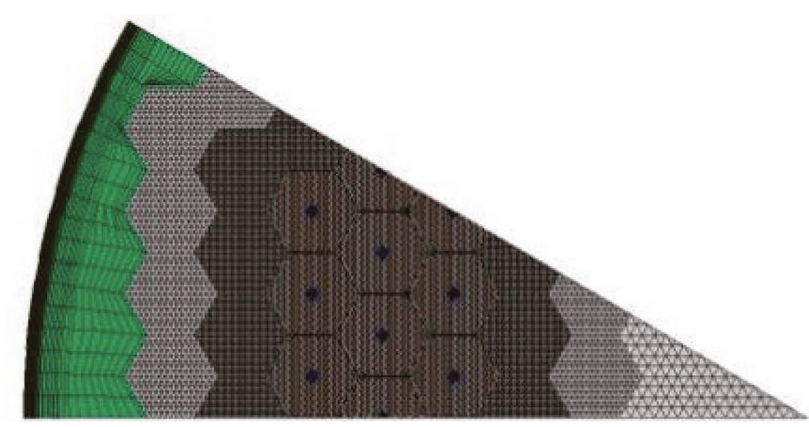

Fig. 18. HTR Core with 1/12 Symmetry Showing the Partition in Regions a set of dedicated modules and the multigroup flux iterators adapted for the calculation of the generalized importance function. [77] The responses can be the reaction rates, reaction rates ratios or bilinear ratios for any reaction type.

- A direct interfacing to the TRIPOLI-4 Monte Carlo code has been implemented in order to pass multigroup cross sections for comparison purposes, or to transmit the interface angular flux or currents for the multigroup albedo calculation, which in turn can be reused by APOLLO2.

- Automatic meshing features can be applied to Cartesian geometries, where either macro-regions for $\mathrm{CP}$ calculations or the number and disposition of depletion zones can be determined according to flux criteria based on a given flux distribution. Hence, a fast, finemesh discrete ordinate calculation can be used to yield a coarser mesh for a CP calculation or to diminish the number of depletion zones for a burnup calculation

- A quadrature module builds on the fly angular quadrature formulas for use with the discrete ordinates and collision probability solvers. [78] This module comprises level symmetric, triangular and product quadratures and has the potential for constructing one-dimensional azimuthal quadratures for finite open angles as well as cyclic angular quadratures for closed rectangles and hexagons. [74]

- Also, a dedicated GUI SILENE has been developed to facilitate geometry description and meshing for regular and unstructured geometries. [79] This graphical interface allows for the use of a library of pre-defined geometries that can then be used as components for larger geometries.

Table 4. 99-group MOC Calculation of a BOL HTGR with 1/12 Symmetry. Comparisons of Normalized Assembly Rates with the TRIPOLI-4 Reference Values. TRIPOLI-4 Standard Deviation (\%) and MOC Relative Error (\%) in Parenthesis

\begin{tabular}{c|c}
\hline TRIPOLI-4 & MOC \\
\hline $1.004(0.09)$ & $0.997(-0.76)$ \\
\hline $0.969(0.09)$ & $0.962(-0.71)$ \\
\hline $1.003(0.09)$ & $0.995(-0.80)$ \\
\hline $0.916(0.13)$ & $0.912(-0.39)$ \\
\hline $0.872(0.09)$ & $0.871(-0.07)$ \\
\hline $0.889(0.09)$ & $0.890(0.15)$ \\
\hline $0.895(0.13)$ & $0.895(0.05)$ \\
\hline $1.036(0.12)$ & $1.041(0.49)$ \\
\hline $1.181(0.08)$ & $1.171(1.09)$ \\
\hline
\end{tabular}




\section{APOLLO2-A, AN INDUSTRIAL APPLICATION OF THE APOLLO2 CODE IN AREVA}

Framatome (former name of AREVA NP reactor manufacturer) first adopted APOLLO2 in 1993 for its industrial applications. The first industrial version, based on V2.5 and named APOLLO2-F, is integrated since then in one of AREVA's PWR Reactor Code Systems currently used for production: the SCIENCE System. [80] Within the SCIENCE System, APOLLO2 is presently used for safety analyses and fuel reload studies for tens of operating reactors. Moreover it has been used for the core design of the EPR ${ }^{\mathrm{TM}}$ Reactor as well as for its safety assessment (FSAR preparation) for the first reactors of this type currently under construction in Finland, France and China.

APOLLO2 as a part of the SCIENCE System is licensed by several Safety Authorities around the world (US, France, China, Belgium, South Africa and Finland). The licensing process is ongoing for other countries and is expected in the future for countries where potential customers are located. A dedicated R\&D program to extend the physical validity range of the code in terms of discharge burn-up, burnable absorber content, etc. is ongoing since years through a collaboration of CEA, EDF and AREVA.

In 2002 AREVA decided a renewal of its neutronics code systems and launched the development of the ARCADIA $^{(B)}$ Reactor Code System. For ARCADIA ${ }^{(B)}$ AREVA has decided to continue to use the APOLLO2 code. This new application will be described in this section.

\subsection{Overview and Capabilities}

AREVA has adapted CEA's APOLLO2 (V2.8) to its industrial needs as a stand-alone neutron transport code as well as cross section generator code. This industrial version, named APOLLO2-A, [81-83] is designed for PWR and BWR lattice physics calculations as part of the new ARCADIA ${ }^{(B)}$ Reactor Code System. [9] The industrial adaptation consists of a layered computational methodology (multi-level scheme) and a flexible input/output software layer. A comprehensive verification and validation base was set up. APOLLO2-A is coupled with AREVA's PWR and BWR 3D core simulators ARTEMIS [84] and MICROBURN-B2. [85] In 2010 a Topical Report for the ARCADIA $^{(B)}$ system, including APOLLO2-A, was submitted to the US NRC (PWR applications and uranium oxide fuel). Licensing will be extended to mixed oxide (MOX) fuel and BWR applications.

APOLLO2-A uses the CEA2005 multi-group library based on JEFF3.1.1, [13] with the fine 281-group SHEM energy mesh by CEA. [15]

The following types of calculations are available with APOLLO2-A:
- Single PWR or BWR fuel assemblies (all types in the market) with various boundary conditions; including large rods, diamond-shaped water hole, etc.

- Color-set: $2 \times 2,3 \times 3, N \times N$ fuel assemblies up to quarter cores, varying water gaps.

- Special cases: 1D-slab calculation for reflector crosssection generation, single fuel cell.

APOLLO2-A provides the capability of computing single state points, as well as complex depletion histories with varying parameters and branch (all standard feedback parameters and user-defined ones) calculations. Restart points can be included at any state point and burn-up value. Small cores or critical experiments (KRITZ, EPICURE, Babcock and Wilcox, etc.) can also be computed through either the single fuel assembly or the color-set type of calculation. Beyond the standard computation mode, APOLLO2-A can be operated in the so-called "reference" mode, enabling a greater input flexibility (rods, moderating structures etc.) and accuracy. This is obtained by using the finest energy and space mesh with no collapsing, homogenization and time-saving strategies.

Thanks to the programmable and modular structure of APOLLO2 kernel, the industrial version was built with a two-layer software structure: a $\mathrm{C}++$ front-end (input/output processor and task-handler, enabling parallelization and complex history depletion schemes); the F77/F95 APOLLO2.8 kernel with extended functionalities driven by a script-like programmable file.

This two-layer structure enables an easy input for industrial users, fast code evolution and maintenance as well as the capability of fine tuning of the physical models and solvers for advanced users.

APOLLO2-A input is based on a user friendly keywordbased file describing various physical objects (ex. rod, assembly, detector, etc.) The results are stored in a treestructured, customizable hierarchical data file (HDF). [86] An embedded generator of input decks for MCNP [87] and TRIPOLI [24, 25] Monte-Carlo codes was developed.

\subsection{Methodology (Computation Scheme)}

In close partnership with CEA, a methodology for industrial PWR, BWR applications based upon the MOC transport solver $[4,88]$ and a multi-level scheme was setup by AREVA. This multi-step approach is an adaptation of the generic flux reconstruction methodology developed by CEA (see Section 5) to the specific needs of AREVA's computation scheme.

Microscopic cross-sections are first processed at 281 groups to treat resonance self-shielding for the main resonant isotopes $(\mathrm{U}, \mathrm{Pu}, \mathrm{Gd}, \mathrm{Er}, \mathrm{Dy}, \mathrm{Ag}$, In, Cd, Hf, $\mathrm{Zr}$, $\mathrm{Fe}, \mathrm{Co}, \mathrm{V}, \mathrm{Rh}$, etc.). The spatial dependency of the selfshielded cross-sections and the Dancoff effect are taken into account explicitly by a 2D CP flux calculation. Then the 2D heterogeneous flux is computed with MOC on a coarser energy mesh (35 groups, optimized for LWR). The required cross-sections are previously collapsed 
through an "intermediate" flux. The main principle behind this multi-step methodology, shown in Fig. 19 is the decoupling of the flux energy description from the spatial description.

- First, fine 281-energy group flux calculations are performed on simplified geometries with the CPM solver (1st level). The set of CP fluxes is accurate from the energy point of view, but less accurate for the assembly-range spatial coupling.

- To enhance spatial coupling at the full assembly scale, a second flux calculation (2nd level) is performed with the IDT solver $[89,90]$ on a $2 \mathrm{D}$, homogeneous cell geometry with 44-group cross-sections. These cross-sections are obtained by collapsing the selfshielded cross-sections with the CP fluxes.

- The two fluxes from 1st and 2nd level are combined using a reconstruction process [58] to yield a 281-group flux with a cell-scale spatial accuracy over the whole assembly geometry. This is used to collapse the crosssections to 35 groups for usage by the MOC solver.

- The MOC flux calculation is then carried out on a very fine spatial grid (thousands of meshes) representing the true heterogeneous geometry. Examples for BWR fuel assemblies are provided in Fig. 20.

- The MOC flux is combined, with a critical buckling correction, with the 281-group weighting flux to obtain the final result: a 2D flux which is very accurate over the full domain (fuel assembly or color-set) both in space (MOC mesh) and energy (281 groups). It is used for depletion (pin- and ring-wise) and output.

The depletion (fuel, burnable detector and absorbers) is performed with an extended isotope chain (26 actinides and $131 \mathrm{FP}$ ). Zero power decay calculations are supported (shutdown cooling). A detailed energy deposition methodology is implemented for pin-by-pin power distributions: besides local energy deposition in the pellet (by fission product kinetic energy and beta energy), neutron kinetic energy loss by slowing-down and gamma energy

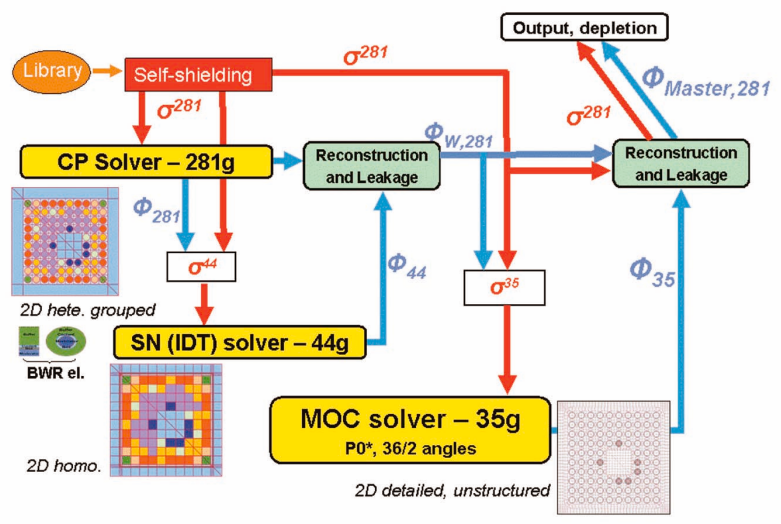

Fig. 19. Multi-level Computation Scheme of APOLLO2-A transport are accounted for. A gamma transport calculation (with neutron-induced sources) is performed with the MOC solver to compute the gamma contribution to the detector response as well as for the energy deposition.

The validation strategy of APOLLO2-A is based both on extensive code-to-code benchmarking (against MonteCarlo calculations with consistent libraries) and comparisons to experimental results (critical experiments and spent fuel analyses).

\subsection{Monte-Carlo Benchmarks}

APOLLO2-A is benchmarked against MCNP at zero burn-up on a wide range of fuel assembly types and physical conditions, representative of existing LWR reactors in operating and accident conditions: more than 450 cases are included, as described below. Each MCNP calculation is a single fuel assembly calculation with reflective boundary conditions. The k-infinity as well as the pin-by-pin fission rate distributions have been compared for each calculation.

The MCNP test base [83] is composed of two separate sets of cases for PWR and BWR fuel assemblies. For PWR fuel assemblies, lattices from $14 \times 14$ to $18 \times 18$, four kinds of spacer grids with various combinations of Inconel, stainless steel and Zyrcaloy content are considered. For uranium oxide fuel, three different U235 enrichment values are included: $1.8,3.7$ and $4.95 \%$. For MOX fuel, three different Plutonium vectors based on fuel reprocessing data are treated, two different maximum plutonium mass content values (5.95 and 12\%) and two realistic three-zone rod spatial distributions. A configuration with reprocessed uranium is also considered. For burnable absorbers, gadolinium-doped rods with six different configurations (from $2 \%$ to $10 \%$ gadolinium oxide mass fraction, including one or two values per assembly) are considered. Two configurations with dysprosium- and erbium-doped fuel are also used. 
Special absorbers are also considered: three configurations with IFBA (integral fuel burnable absorber), three withWABA (wet annular burnable absorber) and three with PYREX $\left.{ }^{(}\right)$rods with variable number of rods. Cladding materials are Zyrcaloy and $\mathrm{M} 5^{\circledR}$.

To ease the comparisons the test cases are run with the same sets of physical conditions. The choice of the conditions aimed at covering the largest physical domain: in addition to most of the standard operating conditions, some accidental conditions are also tested. It must be noted that not all the geometry and material configurations are tested in all physical conditions, since the number of combinations would be of several thousands: a selection, based on the decoupling of physical affects, was made to reduce the total number of calculations.

For PWR assemblies, fifty-six different cases are tested representing fourteen different physical conditions with four control rod configurations: without absorber rods, with AIC rods, with B4C rods and with hafnium rods. The physical parameters span the following ranges: fuel temperature ( 293.16 to $900 \mathrm{~K}$ with 3 points), boron concentration ( 0 to $2500 \mathrm{ppm}$ with 4 points), moderator density ( 0.2 to $1 \mathrm{gcm}^{-3}$ with 4 points), moderator temperature (293.16 and 600K), isotopic perturbation (two configurations of Xe135 and Sm149). The combinations of such values used for the calculations are those corresponding to the following reactor conditions: hot zero power, hot full power, cold and main steam line break (low moderator density).

For BWR fuel assemblies, most of the existing assemblies in the market are considered: ATRIUM with square moderating rod $(9 \times 9,10 \times 10)$, large moderating rods $(9 \times 9$ and $10 \times 10)$, diamond shape moderating rods and cross $(10 \times 10)$, straight-wings moderating cross $(8 \times$ 8 ), with both simple and reinforced channel box models (10 types). Realistic fuel rod arrangements based on vendors design are considered for both uranium oxide and MOX. Truncated rods configurations are also included for some of the above fuel assembly types. Three different control blade designs are considered: one with vertical rods and two with horizontal rods and vertical slabs, with $\mathrm{B}_{4} \mathrm{C}$ and hafnium absorbers. A total of forty-five different cases are tested representing nine different physical conditions. The physical parameters for BWR cases span in the following ranges: fuel temperature (293.16 to $900 \mathrm{~K}$ with 3 points), boron concentration ( 0 and $2000 \mathrm{ppm}$ ), void (0 to $100 \%$ with 6 points) and moderator temperature (293.16 and 600K). The combinations of such values used for the calculations are those corresponding to hot zero power, hot full power and cold conditions.

The uncertainties $(1 \sigma)$ of the MCNP calculations are low: $\pm 10 \mathrm{pcm}$ for $\mathrm{k}$-infinity and $0.3 \%$ for pin-by-pin fission rates. The average discrepancies in PWR and BWR fuel assembly k-infinity are $-48 \pm 124 \mathrm{pcm}, 42 \pm 325 \mathrm{pcm}$ for uncontrolled and controlled assemblies, respectively. The root mean square (RMS) differences for pin-by-pin fission rates between APOLLO2-A and MCNP are shown in Fig. 21. The differences in fission rate RMS are $0.25 \pm$ $0.10 \%$ and $0.46 \pm 0.18 \%$ for PWR and BWR assemblies,

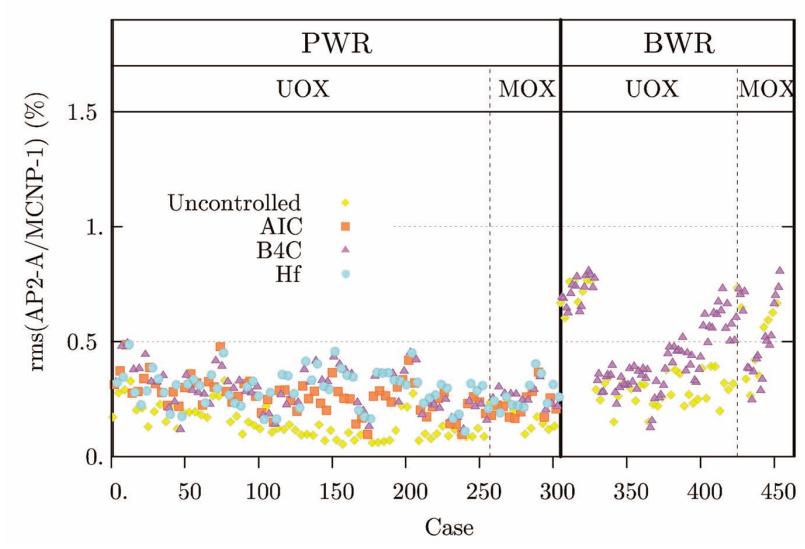

Fig. 21. Relative Difference on Pin-by-pin Fission Rate Rms Between APOLLO2-A and MCNP

Table 5. Critical Experiment Characteristics

\begin{tabular}{c|c|c|c}
\hline Experiment & Enrich. (w/o) & Temp. $\left({ }^{\circ} \mathrm{C}\right)$ & Special Characteristics \\
\hline B\&W & $2.5-4.0$ & 21 & $\begin{array}{c}\text { Absorbers: Pyrex, B4C, AIC, Gd2O3 } \\
(\text { Gd Enrichment }=4.00 \%)\end{array}$ \\
\hline KRITZ-KWU & 3.1 & $20-245$ & Temperature Variation \\
\hline EPICURE & 3.7 & 22 & $\begin{array}{c}\text { Absorbers: Pyrex, AIC - } \\
\text { Void simulated by Al over-cladding }\end{array}$ \\
\hline CAMELEON & 3.5 & 22 & $\begin{array}{c}\text { Absorbers: Hf, Gd2O3 (Gd } \\
\text { Enrichment }=3 \% \text { with 5.1 w/o } \\
\text { U2235 and 7\% with } 0.25 \text { w/o U235) }\end{array}$ \\
\hline
\end{tabular}


respectively. The entire set of results shows the excellent agreement between the two codes.

\subsection{Critical Experiments}

The analyzed experiments come from several international programs, including Babcock and Wilcox $(B \& W)$ in the US, KRITZ-KWU in Sweden, and two experimental programs - EPICURE and CAMELEON from CEA. These critical experiments are UO2-fueled experimental reactors which were selected to support the licensing of the ARCADIA ${ }^{B}$ chain in the US. The general characteristics of the experiments are given in Table 5. A total of 43 configurations were analyzed for reactivity.

The mean deviation of the k-eff among all the configurations is $100 \pm 150 \mathrm{pcm}$ at $1 \sigma$. Only two configurations showed more than $250 \mathrm{pcm}$ of discrepancy. CEA has determined that the uncertainty, in pcm, of its critical experiments, including measurement and modeling, is between 300 and $400 \mathrm{pcm}(1 \sigma)$. These results clearly show a good agreement well within the overall uncertainty. All of the results for the fission rate distributions fall within $2 \sigma$ of the reported measurement uncertainty $(2 \%$ at $2 \sigma$ ).

\subsection{Spent Fuel Analyses}

The isotopic inventory of the spent fuel calculated by APOLLO2-A is compared with the measured compositions from chemical analyses performed on samples irradiated in experimental and power reactors. The samples come from several power plants in Germany and France as well as a CEA experimental reactor, and cover a large range of fuel types, including UOX, Enriched Reprocessed Uranium (ERU), UO2-Gd2O3 and MOX, as well as a large range of burn-up levels, from 3 to $71 \mathrm{GWd} / \mathrm{t}$. The UOX, ERU and UO2-Gd2O3 cases were computed with single-assembly calculations, whereas those cases involving MOX were computed using the color-set capability, in order to account for the spectral effect of neighboring assemblies. Table 6 presents the general characteristics of the spent fuel analysis experiments.

The results for the spent fuel analyses, presented in Fig. 22, show that for most of the isotopes and burn-up values, APOLLO2-A predicts the isotopic inventory within $5 \%$ of the measurement. More than $95 \%$ of the results for isotopes of interest are within $2 \sigma$ of the total reported uncertainties - up to $7 \%(1 \sigma)$ depending on isotopes and burn-up.

\section{APOLLO2 IN EDF}

Electricité de France (EDF) is presently responsible for the operation of nuclear power plants in France, involving 58 PWRs and for the design of the next generation EPR in Flamanville and Penly. In order to deal with PWR fuel management and core safety analyses, EDF has developed and validated its own core calculation chain, CASSIOPEE which integrates the code APOLLO2. [10]

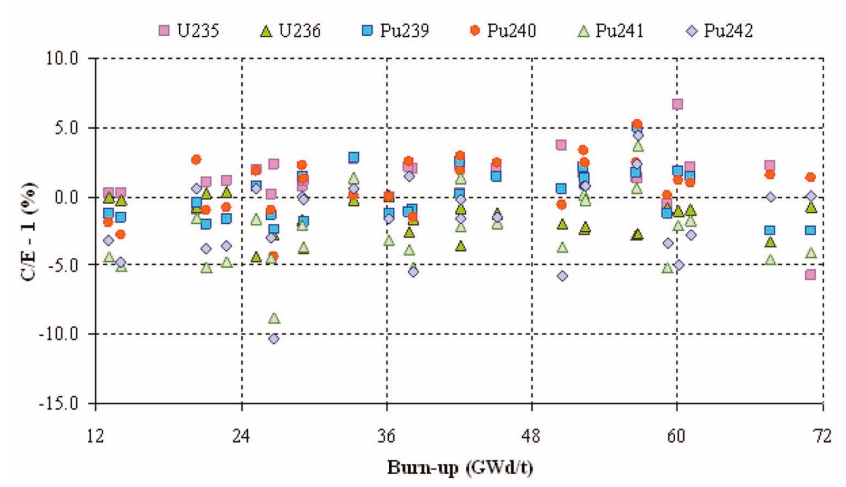

Fig. 22. Results of Spent Fuel Analyses

Table 6. Isotopic Burn-up Analysis Experiments

\begin{tabular}{c|c|c|c}
\hline Experiment & Fuel Type & \# Samples & Burn-up Range \\
\hline Bugey 3 & UOX: 3.1\% U235 Enrichment & 1 & $20 \mathrm{GWd} / \mathrm{t}$ \\
\hline Gravelines 2\&3 & UOX: 4.5\% U235 Enrichment & 7 & $26-61 \mathrm{GWd} / \mathrm{t}$ \\
\hline \multirow{2}{*}{ Malibu Program } & UOX: 4.3\% U235 Enrichment & 1 & $71 \mathrm{GWd} / \mathrm{t}$ \\
\cline { 2 - 5 } & MOX: 8.1\% Plutonium & 1 & $68 \mathrm{GWd} / \mathrm{t}$ \\
\hline Cruas 4 & ERU: 3.1\% U235, 1.2\% U236 & 6 & $13-36 \mathrm{GWd} / \mathrm{t}$ \\
\hline Gedeon 1 Program & UOX: 3.25\% Enrichment $\mathrm{Gd}_{2} \mathrm{O}_{3}: 5 \%$ & 12 & $3.5-8.5 \mathrm{GWd} / \mathrm{t}$ \\
\hline Gedeon 2 Program & UOX: 0.2\% Enrichment Gd $\mathrm{O}_{3}: 8 \%$ & $71.8 \mathrm{GWd} / \mathrm{t}$ \\
\hline Saint Laurent B1 & MOX: 2.9-5.6\% Plutonium & 7 & $25-45 \mathrm{GWd} / \mathrm{t}$ \\
\hline Dampierre 2 & MOX: 6.7\% Plutonium & 4 & $52-57 \mathrm{GWd} / \mathrm{t}$ \\
\hline
\end{tabular}


This section briefly reviews the use of APOLLO2 transport code at EDF, it mainly focuses on the production, conception and $\mathrm{R} \& \mathrm{D}$ aspects.

\subsection{APOLL02.5: the Industrial Lattice Code in EDF}

The EDF core calculation chain, CASSIOPEE, includes two main components:

- The GAB package, which aims to generate the neutronic libraries of assembly 2-group cross-sections using the CEA deterministic transport code APOLLO2.5.

- The COCCINELLE core calculation code, which solves the diffusion equation with two energy groups and homogeneous assemblies.

The GAB package ("Library Automatic Generator codes system") has been developed by EDF to automate lattice physics calculations (Fig. 23). GAB implements the industrial calculation scheme by executing about one thousand calculations accounting for burn-up, feedback parameters change and geometry changes with insertion of control rods.

Transport calculations are performed using the 99group energy cross section library CEA 93 release 6 and the Collision Probability Method (CPM) with a multicell simplified geometry. Using the transport flux as a weighting function, spatial homogenization and energy collapsing are performed within GAB.

\subsection{APOLLO2.8: the Future Lattice Code in EDF}

EDF R\&D is currently developing a new calculation scheme aimed at calculating the safety parameters of PWRs. EDF main motivation to renew its calculation scheme is driven by a need to further increase the accuracy of its core calculations. This will be achieved by using state of the art neutronic codes and methods and the most recent nuclear data.
The future EDF calculation scheme is based on the classical 2-step approach. The first step consists in computing few-group cross sections libraries thanks to assembly calculations performed with CEA's deterministic transport code APOLLO2.8. The second step deals with whole core $3 \mathrm{D}$ calculations using the data computed at the first stage. EDF's next generation core code is the code COCAGNE [91] developed at EDF R\&D.

\subsubsection{The REL2005 Scheme}

The transport calculations of the scheme are based on the deterministic LWR assembly calculation scheme REL2005 [17] which has been developed at CEA. The 2D lattice transport deterministic code used for this purpose is APOLLO2.8. The multigroup cross sections library associated with the REL2005 scheme uses the SHEM 281-group energy mesh. [15] The isotope cross sections gathered in the multigroup library used by APOLLO2 are mainly from the JEFF3.1.1 punctual evaluations. [13] The assembly calculation stage consists in solving the transport equation with a fine space and energy discretization. In order to obtain a good trade-off between accuracy and calculation speed, the assembly calculation is divided in two phases, as shown in Fig. 24:

- The first one consists of a fine flux calculation using a multicell collision probability approach. The 281group flux so computed is then used as a weight function to perform cross section energy collapsing (from 281 groups to 26 groups).

- The second phase is a detailed spatial calculation where the geometry is explicitly treated using the optimized "windmill mesh." An example of this mesh for LWR fuel assemblies is given in Fig. 25. The solver used is based on the method of characteristics.
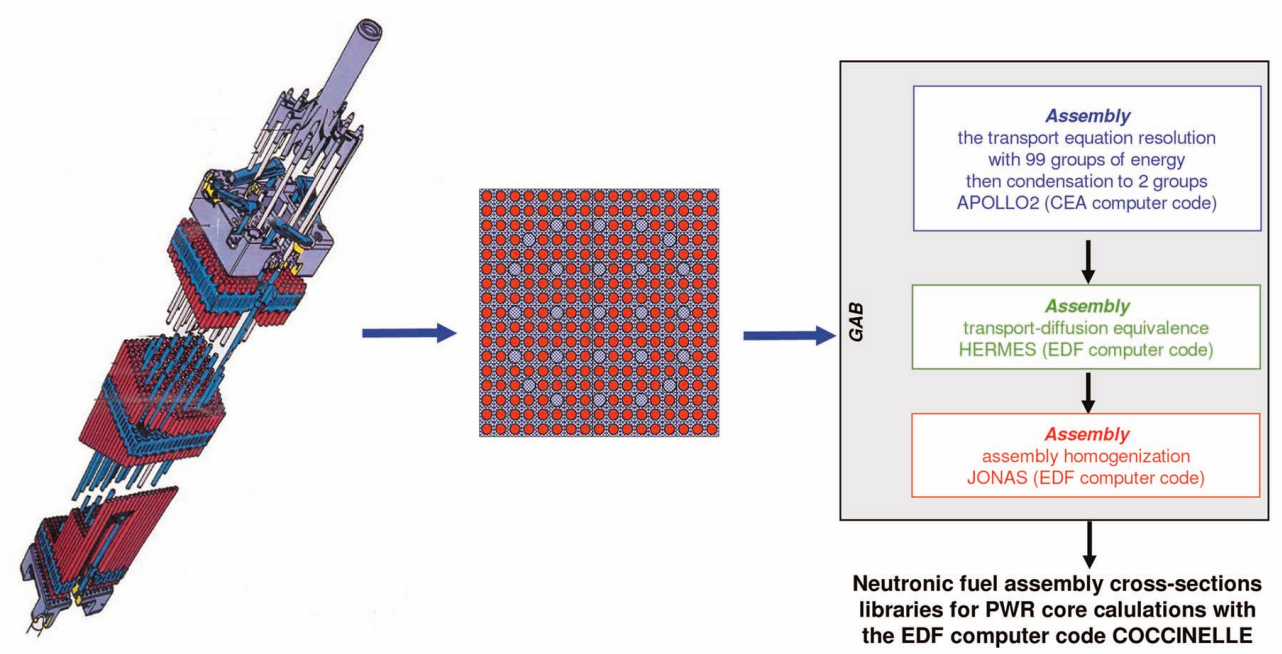

Fig. 23. Generation of the Neutronic Assembly Libraries with the GAB Package 


\subsubsection{Assembly Calculation for the EDF Core Calculation Scheme}

The resulting 26-group flux is first used as a weighting function in the final collapsing of the cross sections from 26 to 2 energy groups. Additionally, a homogenization phase yields equivalent cross sections that may be used by the core code. Depending on the core calculation type, heterogeneous or homogeneous, the homogenized geometry is either a lattice of homogeneous cells describing the pin-by-pin assembly structure or alternatively the whole assembly. Cross sections feedbacks are calculated by interpolating in the multi-parameter cross sections tables of the cross-section library.

The main benefits of APOLLO2.8/REL2005 scheme are an improved cross section library with a refined energy discretization and the MOC transport solver used on a refined spatial mesh, while the calculation time still complies with industrial constraints.

\subsubsection{APOLLO2 Verification \& Validation}

The new optimized calculation scheme REL2005 has been verified by CEA $[17,19]$ for all types of assemblies used in EDF PWRs, UOX and MOX assemblies, with or without burnable poisons and/or with or without inserted control rods. This verification is based on the comparison of APOLLO2.8/REL2005 calculation results against continuous-energy Monte Carlo TRIPOLI-4 [24, 25] reference calculation. As an example, this comparison for specific PWR benchmarks shows the ability of the deterministic tool to calculate UOX assemblies poisoned with gadolinium within $200 \mathrm{pcm}$ accuracy on reactivity and $1 \%$ on the pin-by-pin power distribution. Further verification work will be completed against startup tests and flux map measurements in EDF PWR fleet.

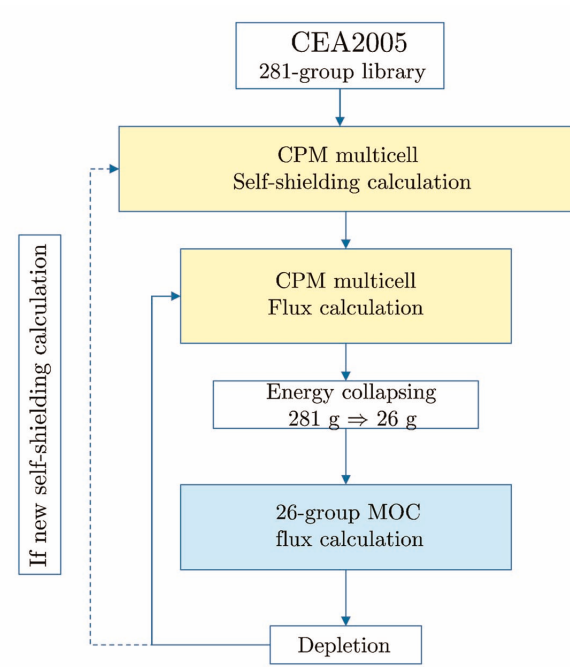

Fig. 24. New APOLLO2.8 LWR Calculation Scheme REL2005
Currently, the package APOLLO2.8-MOC/CEA2005SHEM is undergoing a large validation program with the CEA. Calculation results performed by the CEA with REL2005 scheme show good agreements with the experiments:

- Reactivity is slightly over predicted for the UO2 and mixed MOX-UO2 configurations: $510 \mathrm{pcm} \pm 600 \mathrm{pcm}$ $(2 \sigma) .[92]$

- The results obtained on pin-by-pin fission rates for UO2 and MOX assemblies are also very satisfactory, especially near the interface: $\pm 1.5 \% \pm 1.6 \%(2 \sigma)$. [92]

- Absorber worths are overestimated by $1.2 \%$ to $2.7 \%$ when proper 3D correction, taking into account axial buckling variation, is applied on the $2 \mathrm{D} \mathrm{k}_{\text {eff }}$. [93]

- Pin-by-pin power at the core/baffle interface is slightly overestimated by about 2 to $3 \%$. [94]

- The reactivity worth of large partial void, typically $60 \%$ void, is slightly overestimated by $+1.0 \%$, that lies within the $\pm 1 \%(1 \sigma)$ experimental uncertainty margin. The measured radial fission maps are particularly well predicted, even in the $60-100 \%$ high void fraction configurations. [95]

\subsection{APOLLO2.8 Used as a Reference Tool}

APOLLO2.8 is also extensively used as a reference tool facilitating the optimization of the nuclear core code. It provides transport flux and reaction rates solutions for fuel assemblies and assembly colorsets that are used for verification in the following procedures: transport-diffusion equivalence, [96] pin power reconstruction (Fig. 26), [97] and microscopic depletion. [98]

After an ongoing validation phase, for which very satisfactory results have been obtained to date, these new models will be implemented in the future EDF core calculation chain.

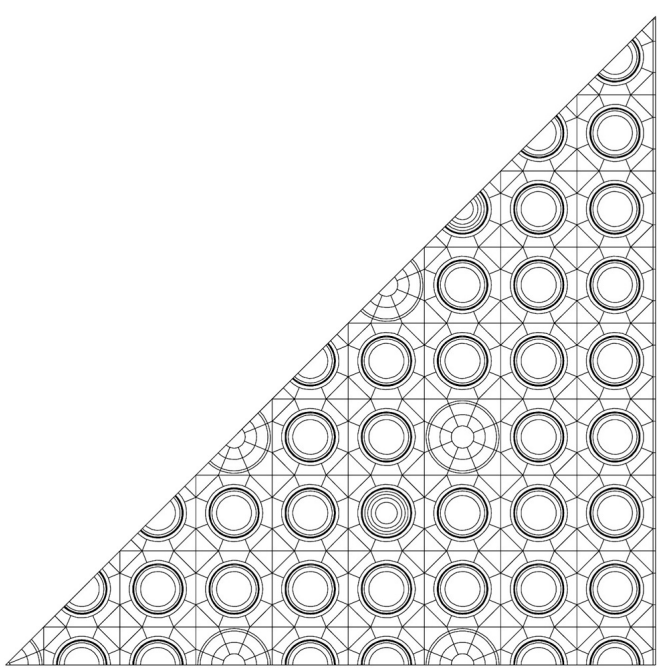

Fig. 25. Optimized "Windmill Mesh" for LWR Assemblies 


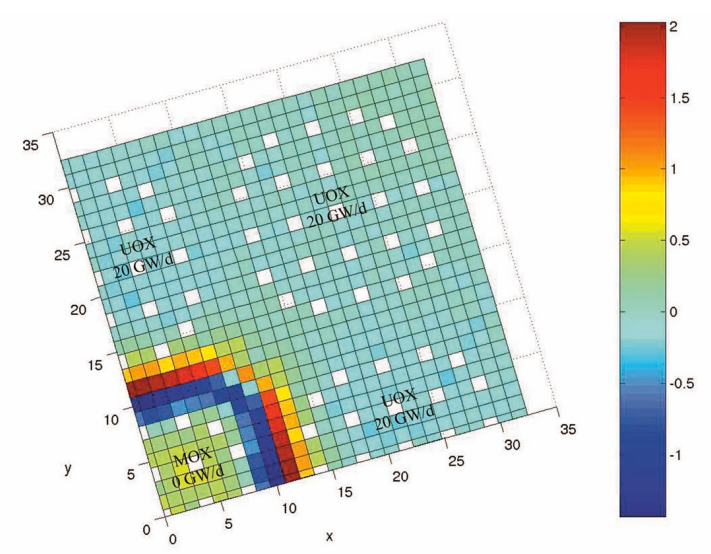

Fig. 26. Fission Rate Relative Distribution Discrepancies (\%) for MOX/UOX Configuration Between COCAGNE and APOLLO2/REL2005

\section{CONCLUSIONS}

With the incorporation of new homogenization techniques (resonance mixture and transfer matrix selfshielding), flux reconstruction methods, new or improved flux solvers (CPM for RZ geometry, heterogeneous cells for short MOC and the linear-surface scheme for long MOC), improved acceleration techniques $\left(\mathrm{DP}_{1}\right)$, that are also applied to thermal and external iterations, and a number of sophisticated modules and tools to help user calculations, the latest version of the APOLLO2 code has reached a degree of maturity which makes it an excellent tool for industrial applications.

New developments are written in FORTRAN90 and tested in independent mock-ups (IDT, TDT) before their coupling, via an interface, with the APOLLO2 code. A 'developers' version, based on the latest APOLLO2 one, allows the development team of the APOLLO2 code to create and test new methodologies which are later integrated in the official version. Methods for reflector homogenization, for both $\mathrm{P}_{N}$ transport (for applications to simplified $\mathrm{P}_{N}$ core calculations) and diffusion, [99, 100] have been incorporated in the developers' version, as well as a stochastic scheme for pebble-bed core calculations based on dynamic transport homogenization. [101] Analyses have also been carried out for higher-order methods of characteristics [102] as well as for non-linear acceleration schemes. [103, 104]

New developments that will be incorporated in the next release of the code comprise an updated angularquadrature module with Galerkin angular quadratures [105] and extended capabilities for the extraction of cell-based surfaces-averaged fluxes and partial currents so as to generate flux discontinuity factors for diffusion and simplified $\mathrm{P}_{N}$ core calculations. [42]

At the time of its conception (the first $\mathrm{k}_{\text {eff }}$ was obtained in 1984) the in-house software, [29, 30] allowing for dynamic allocation, data structuring and archive of calculations for future re-starts, made possible the adoption of a modular architecture for the APOLLO2 code and the use of an user's command language, [31] which offered an enormous advantage for users and developers. However, the advent of commercial languages with dynamic allocation and object-oriented programming have made those earlier tools somewhat obsolete and presently made difficult drastic changes in the code. This is one of the reasons that have prompted the Commissariat à l'Energie Atomique et aux Energies Alternatives to start the development of a new software, the APOLLO3 code. [106, 107] This new code, written in $\mathrm{C}++$ and FORTRAN2003 with an object-oriented architecture compatible with massive parallel computers, is meant to include the existing capabilities of APOLLO2 and the core codes CRONOS [108] and ERANOS [109] within a single platform, incorporating the experience and know how of the developers and users of these three codes. APOLLO3 is fully compatible with the Monte Carlo code TRIPOLI-4, allowing calculations based on the complementary use of both software packages, as well as with the nuclear-data treatment code GALILEE [26] and the depletion software package MENDEL, [110] with which it shares common functionalities.

At the present time the code already disposes of a 3D linear discontinuous finite element method for axial geometries with an unstructured XY cross section generated by an automatic mesh generator [106] as well as $3 \mathrm{D}$ discrete-ordinates methods [48] and a $3 \mathrm{D} \mathrm{SP}_{N}$ solver. This new software package will allow present-style twostep core calculations to be done in a single run as well as an easy implementation of dynamic homogenization core calculation schemes. [101, 111] By using parallel architectures, three-dimensional transport calculations are possible for assembly, clusters and, with collapsed cross sections, for homogenized or pin-by-pin full core calculations. The analysis of these techniques in under progress for the short and long MOC methods.

Nevertheless, APOLLO2 will remain for long time in use and new developments will be added to the code as needed. Long live APOLLO2!

\section{Acknowledgements}

The APOLLO2 code is the results of a long interaction between developers and users and has benefited from the experience and work of too many individuals to be cited here. The authors express their thanks to all the pass and present conceptors, developers, project managers and, especially, the users of APOLLO2 for their contributions which have made possible the success of the code. The CEA authors are indebted to Areva-NP and EDF for their financial support as well to the AREVA and EDF authors for their contributions in Sections 10 and 11. One of the authors (RS) thanks Anna K. for help with the graphics. Another author (EM) thanks S. Thareau, P. Magat, F. 
Clement, M. Schneider, J. Marten, S. Misu, A. Cheng and the many colleagues of AREVA NP Paris, Erlangen and Lynchburg who participated in the deployment of APOLLO2-A. The material in sections 5 and 6 has been partially reproduced from references [58] and [60] with permission from the Canadian Nuclear Society. Figure 10 and Table 2 have been reproduced from figure 8 and a part of Table 4 in reference [62] with permission from the American Nuclear Society (copyright 2009 by the American Nuclear Society, La Grange Park, Illinois). Figures 12 and 13 have been reproduced from figures 7 (right side only) and 8 in reference [71] with permission from the American Nuclear Society (copyright 2009 by the American Nuclear Society, La Grange Park, Illinois). Figures 21 and 22 and tables 5 and 6 have been partially reproduced from figures 2 and 5 and tables I and III in reference [83] with permission from the American Nuclear Society (copyright 2010 by the American Nuclear Society, La Grange Park, Illinois).

\section{REFERENCES}

[1 ] S. Rauck, R. Sanchez, I. Zmijarevic and M. Nobile, "A Multigroup Albedo Method for Transport Calculations: Application to the Orphée Core," Nucl. Sci. Eng. 135, 73 (2000).

[2] G. Willermoz, A. Aggery, D. Blanchet, S. Cathalau, C. Chichoux, J. Di-Salvo, C. Döderlein, D. Gallo, F. Gaudier, N. Huot, S. Loubière, B. Noël and H. Servière, "HORUS3D code package development and validation for the JHR modeling," Proc. Int. Conf. The Physics of Fuel Cycles and Advanced Nuclear Systems (PHYSOR 2004), Chicago, IL, USA, April 25-29, 2004.

[ 3 ] N. Huot, A. Aggery, D. Blanchet, C. D’Aletto, J. Di Salvo, C. Döderlein, P. Sireta and G. Willermoz, "The JHR Neutronics Calculation Scheme Based on the Method of Characteristics," Proc. Int. Top. Mtg. on Mathematics and Computation, Supercomputing, Reactor Physics and Nuclear and Biological Applications (M\&C 2005), Avignon, France, Sept 12-15, 2005.

[4] R. Sanchez and A. Chetaine, "A synthetic acceleration for a two-dimensional characteristic method in unstructured meshes," Nucl. Sci. Eng., 136, 122 (2000).

[ 5 ] G. Campioni and B. Desbrière, "HFR advanced computation's models," Proc. Int. Conf. on Physics of Reactors, "Nuclear Power: A Sustainable Resource," (PHYSOR 2008), Interlaken, Switzerland, Sept. 14-19, 2008.

[6] A Tsilanzara, C.M. Diop, B. Nimal et al., "DARWIN: an Evolution Code System for a Large Range of Applications," Nucl. Sci. and Technology, 37, Part SUPP/1, 845, (2000).

[ 7 ] B. Roque, N. Thiollay, P. Marimbeau, A. Barreau, A. Tsilanzara, C. Garzenne and F. Marcel, "Experimental validation of the code system DARWIN for spent fuel isotopic predictions in fuel cycle applications," Proc. Int. Conf. on Reactor Physics Safety and High-Performance Computing (PHYSOR 2002), Seoul, Korea, 7-10 October, 2002.

[ 8 ] J.-M. Gomit, E. Létang, C. Fedon-Magnaud, C. Diop and J.P. Grouiller, "CRISTAL V1: Criticality Package for Burnup Credit Calculation," Proc. of the NCSD 2005 Meeting on Integrating Criticality Safety into the Resurgence of Nuclear Power, Knoxville, TN, USA, September 18-22, 2005.

[9] F. Curca-Tivig, S. Merk, A. Pautz, S. Thareau, "ARCADIA ${ }^{\circledR}$
- A new generation of coupled neutronics / core thermalhydraulics code system at AREVA NP," Proc. Int. Conf. LWR Fuel Performance Meeting, San Francisco, CA, USA, Sept., 2007.

[10] A. Santamarina, C. Collignon, C. Garat, "French Calculation Schemes for Light Water Reactor Analysis," Proc. Int. Conf. on the Physics of Fuel Cycles and Advanced Nuclear Systems (PHYSOR2004), Chicago, IL, USA, April 25-29, 2004.

[11] D.G. Cacuci, J.M. Aragonés, D. Bestion, P. Coddington, L. Dada and C. Chauliac, "NURESIM: A European Platform for Nuclear Reactor Simulation," Proc. FISA 2006: EU Research and Training in Reactor Systems, European Commission, Luxembourg, March 13-16, 2006.

[12] G.B. Bruna, F. Dubois, F. Fouquet, J-C. Le Pallec, E. Richebois, E. Hourcade, C. Poinot-Salanon and E. Royer, "HEMERA: a 3D coupled core-plant system for accidental reactor transient simulation," Proc. ICAPP seventh Int. Conf. on Advances in Nuclear Power Plants, Nice, France, May 13-18, 2007.

[13] A. Santamarina, D. Bernard, P. Blaise, M. Coste, A. Courcelle, T.D. Huynh, C. Jouanne, P. Leconte, O. Litaize, S. Mengelle, G. Noguère, J-M. Ruggieri, O. Sérot, J. Tommasi, C. VaglioGaudard, J-F. Vidal, "The JEFF-3.1.1 Nuclear Data Library, JEFF Report 22, Validation Results from JEF-2.2 to JEFF3.1.1," NEA No. 6807, OECD/NEA Edition 2009.

[14] C. J. Dean, R. J. Perry, "Weighting spectra for the Xmas group scheme," Winfrith Technology Center, 1990.

[15] N. Hfaiedh, A. Santamarina, "Determination of the optimised SHEM mesh for transport calculation," Proc. Int. Top. Mtg. on Mathematics and Computation, Supercomputing, Reactor Physics and Nuclear and Biological Applications (M\&C2005), Avignon, France, Sept 12-15, 2005.

[16] A. Santamarina, N. Hfaiedh, R. Le Tellier, V. Marotte, S. Misu, A. Sargeni, C. Vaglio-Gaudard, I. Zmijarevic, "Advanced neutronics tools for BWR design calculations," Nucl. Eng. and Design 238, 1965 (2008).

[17] J-F. Vidal, R. Tran, O. Litaize, D. Bernard, A. Santamarina, C. Vaglio-Gaudard, "New modelling of LWR assemblies using the APOLLO2 code package," Proc. Joint Int. Top. Mtg. on Mathematics \& Computation and Supercomputing in Nuclear Applications (M\&C + SNA 2007), Monterey, CA, USA, April 15-19, 2007.

[18] A. Santamarina, C. Chabert, A. Courcelle, O. Litaize, G. Willermoz, D. Biron, L. Daudin, "Qualification of the APOLLO2.5/CEA93.V6 code for UOX and MOX fuelled PWRs," Proc. Int. Conf. on Reactor Physics Safety and High-Performance Computing (PHYSOR 2002), Seoul, Korea, 7-10 October, 2002.

[19] A. Santamarina, D. Bernard, P. Blaise, L. Erradi, P. Leconte, R. Le Tellier, C. Vaglio-Gaudard, J-F. Vidal, APOLLO2.8: a validated code package for PWR neutronics calculations," Proc. Int. Conf. on Advances in Nuclear Fuel Management (ANFM-IV), Hilton Head Island, SC, USA, April 12-15, 2009.

[20] O. Litaize, A. Santamarina, C. Chabert, "Analysis of the Mistral Experiment with APOLLO2. Qualification of neutronic Parameters of UOX and MOX Cores," Proc. Int. Conf. on Reactor Physics Safety and High-Performance Computing (PHYSOR2002), Seoul, Korea, 7-10 October, 2002.

[21] A. Santamarina, C. Vaglio-Gaudard, P. Blaise, N. Huot, S. Thareau, O. Litaize, N. Thiollay, J-F. Vidal, "The PERLE Experiment for the qualification of EPR heavy reflector," Proc. Int. Conf. on Physics of Reactors, "Nuclear Power: A 
Sustainable Resource," (PHYSOR 2008), Interlaken, Switzerland, Sept. 14.19, 2008.

[22] P. Leconte, J-F. Vidal, D. Bernard, A. Santamarina, R. Eschbach, "Qualification of the APOLLO2.8 code package for the calculation of the fuel inventory and reactivity loss of UOx spent fuels in BWRs," Ann. Nucl. Energy 36, 362 (2009).

[23] L. Mondelain, I. Zmijarevic, J-M. Do and V. Bellanger, "Use of APOLLO2 for BWR Assembly Analysis," Proc. Joint Int. Top. Mtg. on Mathematics \& Computation and Supercomputing in Nuclear Applications $(M \& C+S N A$ 2007), Monterey, CA, USA, April 15-19, 2007.

[24] C.M. Diop, O. Petit, E. Dumonteil, F-X. Hugot, Y.K. Lee, A. Mazzolo and J-C. Trama, "Review of the TRIPOLI-4 Monte Carlo Transport Code," Proc. of the Int. Cong. on Advanced in Nuclear Power Plant (ICAPP 2007), Nice Acropolis, France, May 13-18, 2007.

[25] E. Dumonteil, F.X. Hugot, C. Jouanne, Y.K. Lee, F. Malvagi, A. Mazzolo, O. Petit, J-C. Trama, C. Diop, "An Overview on the Monte Carlo Particle Transport Code," Advanced Methods, Codes, and Benchmarking of the NURESIM Platform, Proc. of the ANS 2007 Winter Meeting, Washington, D.C., USA, November 11-15, 2007.

[26] M. Coste-Delclaux, "GALILEE: A Nuclear Data Processing System for Transport, Depletion and Shielding Codes", Proc. Int. Conf. on Physics of Reactors, "Nuclear Power: A Sustainable Resource," (PHYSOR 2008), Interlaken, Switzerland, Sept. 14.19, 2008.

[27] A. Kavenoky, “APOLLO: A general code for transport, slowing-down and thermalization calculations in heterogeneous media," Conference 73044 P1, Ann Arbor, Michigan, USA, April 9-11, 1973.

[28] A. Kavenoky and R. Sanchez, "The APOLLO assembly spectrum code," ANS Int. Top. Mtg. on Advances in Reactor Physics, Mathematics and Computations, Paris, France, 1987.

[29] A. Kavenoky and M.F. Robeau, "Un système d'aide à la programmation scientifique ARIANE," Prémière Colloque de Génie Lgiciel, Paris, France, June 8-10, 1982.

[30] M.F. Robeau, Commissariat à l'Energie Atomique, Personal Communication, 1986.

[31] M.F. Robeau and P. Verpeaux, Commissariat à l'Energie Atomique, Personal Communication, 1986.

[32] R. Sanchez, J. Mondot, Z. Stankovski, A. Cossic and I. Zmijarevic, "APOLLO II: A user-oriented, portable, modular code for multigroup transport assembly calculations," Nucl. Sci. Eng., 100, 352 (1988).

[33] S. Loubière, R. Sanchez, M. Coste, A. Hebert, Z. Stankovski, C. Van Der Gucht and I.Zmijarevic, "APOLLO II Twelve Years Later," Proc. Int. Conf. on Mathematics and Computation, Reactor Physics and Environmental Analysis in Nuclear Applications (M\&C'99), Madrid, Spain, Sept. 27-30, 1999.

[34] "APOLLO2 workshop," Int. Top. Mtg. on Mathematics and Computation, Supercomputing, Reactor Physics and Nuclear and Biological Applications (M\&C 2005), Avignon, France, Sept 12-15, 2005.

[35] "APOLLO2 and TRIPOLI-4 workshop," Joint Int. Top. Mtg. on Mathematics \& Computation and Supercomputing in Nuclear Applications (M\&C + SNA 2007), Monterey, CA, USA, April 15-19, 2007.

[36] In "Workshop on Transport Methods for Reactor Core Calculations ," Proc. Int. Conf. on Advances in Reactor Physics to Power the Nuclear Renaissance (PHYSOR 2010),
Pittsburgh, PA, USA, May. 9-14, 2010.

[37] R. Sanchez and J. Mondot, "A model for calculating multigroup self-shielded cross sections for a mixture of resonant absorbers in heterogeneous media," Proc. Mtg. on Advances in Reactor Physics and Radiation Protection and Shielding, Chicago, IL, USA, Sept. 17-19, 1984.

[38] R. Sanchez, M. Coste, Z. Stankovski, C. Van der Gucht, "Models for multi-group self-shielded cross-sections calculations in the code APOLLO2," Proc. Int. Conf. on the Physics of Reactors: Operation, Design and Computation, Marseille, France, 1990.

[39] P. Benoist, I. Petrovic, Z. Stankovski, "Improvements in leakage calculation of nuclear reactor assemblies and consistent definition of cell leakage coefficients by an equivalence procedure," Proc. Int. Conf. on Advances in Mathematics, Computations and Reactor Physics, Pittsburgh, PA, USA, April 28-May 1, 1991.

[40] R. Sanchez, "Une analyse des modèles de fuites," Note CEAN-2803, Commissariat à l'Energie Atomique, 1995 (in French).

[41] A. Hébert and A. Kavenoky, "Development of the SPH homogenization method," ANS/ENS Int. Top. Mtg. on Advances in Mathematical Methods for the Solution of Nuclear Engineering Problems, Munich, Germany, April 27-29, 1981.

[42] R. Sanchez, "Assembly homogenization techniques for core calculations," Progress in Nuclear Energy 51, 14 (2009).

[43] R. Sanchez and G.C. Pomraning, "A statistical analysis of the double heterogeneity problem," Ann. Nucl. Energy, 18, 371 (1991).

[44] A. Hébert, "A collision probability analysis of the doubleheterogeneity problem," Nucl. Sci. Eng., 115, 177 (1993).

[45] R. Sanchez and E. Masiello, "Treatment of the double heterogeneity with the method of characteristics," Proc. Int. Conf. on Reactor Physics Safety and High-Performance Computing (PHYSOR2002), Seoul, Korea, 7-10 October, 2002.

[46] R. Sanchez, "Renormalized treatment of the double heterogeneity with the method of characteristics," Proc. Int. Conf. The Physics of Fuel Cycles and Advanced Nuclear Systems (PHYSOR 2004), Chicago, IL, USA, April 25-29, 2004.

[47] R. Sanchez and M.L. Vergain, "An acceleration procedure for the iterative solution of the flux-current equations in the APOLLO-II code," Proc. Int. Conf. on The Physics of Fuel Cycles and Advanced Nuclear Systems (PHYSOR 2004), Chicago, IL, USA, April 25-29, 2004.

[48] I. Zmijarevic, "Multidimensional Discrete Ordinates Nodal and Characteristics Methods for APOLLO2 Code," Proc. Int. Conf. on Mathematics and Computation, Reactor Physics and Environmental Analysis in Nuclear Applications (M\&C.99), Madrid, Spain, Sept. 27-30, 1999.

[49] S. Perruchot-Triboulet and R. Sanchez, "Décomposition par méthodes perturbatives de la variation de réactivité de deux systèmes," Note CEA N-2817, Commissariat à l'Energie Atomique, 1997 (in French).

[50] R. E. Mac Farlane, D. W. Muir and R. M. Boiscourt, "NJOY99 nuclear data processing system,” Report 12740-M, LANL, 1994.

[51] J.C. Sublet, P. Ribon, M. Coste-Delclaux, "CALENDF-2005: User Manual," Note CEA-R-6131, Nov. 2006 (in French).

[52] P. Mosca, C. Mounier, R. Sanchez and G. Arnaud, “An adaptive energy mesh constructor for multigroup library generation for transport codes," Nucl. Sci. Eng., accepted for publication, June, 2010. 
[53] M. Coste-Delclaux and S. Mengelle, "New resonant mixture self-shielding treatment in the code APOLLO2", Proc. Int. Conf. The Physics of Fuel Cycles and Advanced Nuclear Systems (PHYSOR 2004), Chicago, IL, USA, April 25-29, 2004.

[54] M. Coste-Delclaux, A. Aggery, N. Huot, "New Developments in Resonant Mixture Self-shielding Treatment with APOLLO2 code and Application to Jules Horowitz Reactor Core Calculation," Proc. Int. Top. Mtg. on Mathematics and Computation, Supercomputing, Reactor Physics and Nuclear and Biological Applications (M\&C2005), Avignon, France, Sept 12-15, 2005.

[55] M. Coste, S. Perruchot-Triboulet, C. Van der Gucht, "Selfshielding calculations by APOLLO2 code for fuel pins with a temperature distribution," Proc. ANS Winter Meeting, Saratoga Springs, NY, USA, Oct. 6-10, 1997.

[56] M. Coste-Delclaux, "Recent Progress in Self-shielding Phenomenon Modeling with the APOLLO2 code," Proc. ANS Annual Meeting on Advancing Nuclear Technology for a Greater Tomorrow, Atlanta, GA, USA, June 14-18, 2009.

[57] T. Hazama, "Benchmark calculation of APOLLO2 and SLAROM-UF in fast reactor lattice," Report CEA-R-6223, Commissariat à l'Energie Atomique, 2009.

[58] I. Zmijarevic, E. Masiello and R. Sanchez, "Flux reconstruction methods for assembly calculations in the code APOLLO2," Proc. Int. Conf. Advances in Nuclear Analysis and Simulation (PHYSOR 2006), Vancouver B.C., Canada, Sept. 10-14, 2006.

[59] A. Yamamoto et al., "Benchmark problem suite for reactor physics study of LWR next generation fuels," Nucl. Sci. Technol., 39, 900 (2002).

[60] L. Mao, R. Sanchez, I. Zmijarevic and Z. Stankovski, "RZ calculations for selfshielded multigroup cross sections," Proc. Int. Conf. Advances in Nuclear Analysis and Simulation (PHYSOR 2006), Vancouver B.C., Canada, Sept. 10-14, 2006.

[61] E. Masiello, I. Zmijarevic, "Short Characteristics Method for Two Dimensional Heterogeneous Cartesian Cells," Proc. Int. Conf. Advances in Nuclear Analysis and Simulation (PHYSOR 2006), Vancouver B.C., Canada, Sept. 10-14, 2006.

[62] E. Masiello, R. Sanchez and I. Zmijarevic, "New Numerical Solution with the Method of Short Characteristics for 2-D Heterogeneous Cartesian Cells in the APOLLO2 Code: Numerical Analysis and Tests," Nucl. Sci. Eng. 161, 257 (2009).

[63] A. Yamamoto, "Generalized Coarse-Mesh Rebalance Method for Acceleration of Neutron Transport Calculations," Nucl. Sci. Eng. 151, 274 (2005).

[64] H. Hiruta, D.Y. Anistratov, "Homogenization Method for 2D Low-Order Quasidiffusion Equations for Reactor Core Calculations," Nucl. Sci. Eng. 154, 257 (2009).

[65] “AEN/ NEA Benchmark on deterministic transport calculation without spatial homogenization," Nuclear Science, OECD, Paris (2003).

[66] S. Santandrea and R. Sanchez, "Acceleration Techniques for the characteristics methods in unstructured meshes," Ann. Nucl. Energy, 29, 323, 2002.

[67] R. Barret, M. Berry, T.F. Chan, J. Demmel, J.M. Donato, J. Dongarra, V. Eijkhout, R. Pozo, C. Romine, and H. Van der Vorst, "Templates for the Solution of Linear Systems: Building Blocks for Iterative Methods," SIAM, 1994.

[68] S. Santandrea and R. Sanchez, "Analysis and improvements of the DPN acceleration technique for the method of characteristics in unstructured meshes," Ann. Nucl. Energy, 32, 163-193, 2005.

[69] S. Santandrea, "An Integral Multidomain DPN Operator as Acceleration Tool for the Method of Characteristics in Unstructured Meshes," Nuc. Sci. Eng. 155,1-13, 2006.

[70] S. Santandrea, R. Sanchez and P. Mosca, "A linear surface characteristics scheme for neutron transport in unstructured meshes," Nuc. Sci. Eng. 160, 22, 2008.

[71] S. Santandrea, J.C. Jaboulay, P. Bellier, F. Fevotte, and H. Golfier, "Improvements and validations of the linear surface characteristics scheme," Ann. Nucl. Energy, 36, 46, 2009.

[72] F. Fevotte, S. Santandrea and R. Sanchez, "Advance transverse integration for the method of characteristics," Proc. Joint Int. Top. Mtg. on Mathematics \& Computation and Supercomputing in Nuclear Applications $(M \& C+S N A$ 2007), Monterey, CA, USA, April 15-19, 2007.

[73] A. Koning, R. Forrest, M. Kellett, H. Hneriksson, Y. Rugama, "The JEFF-3.1 Nuclear Data Library," JEFF Report 21, NEA No. 6190, Data Bank, Nov. (2006).

[74] R. Sanchez, L. Mao and S. Santandrea, "Treatment of Boundary conditions in trajectory Based Deterministic Transport Methods," Nucl. Sci. Eng. 140, 23, 2002.

[75] F. Damian, X. Raepsaet, M. Groizard and C. Poinot, "NEPHTIS: core depletion validation relying on 2D transport core calculations with the APOLLO2 code," Proc. Int. Conf. Advances in Nuclear Analysis and Simulation (PHYSOR 2006), Vancouver B.C., Canada, Sept. 10-14, 2006.

[76] F. Damian, X. Raepsaet, S. Santandrea, A. Mazzolo, C. Poinot, J.C. Klein, L. Brault and C. Garat, "GT-MHR Core Modeling: From Reference Modeling Definition in Monte-Carlo Code to Calculation Scheme Validation," Proc. Int. Conf. The Physics of Fuel Cycles and Advanced Nuclear Systems (PHYSOR 2004), Chicago, IL, USA, April 25-29, 2004.

[77] M.L. Williams, "Perturbation theory for nuclear reactor analysis," in CRC Handbook of Nuclear Reactors Calculations, Vol. III. CRC press (1986).

[78] R. Sanchez and I. Zmijarevic, personal communication, 1996.

[79] Z. Stankovski, "Implementation of Component Concept in Silène 2d/3d Pre \& Post Processing GUI," Proc. Joint Int. Top. Mtg. on Mathematics \& Computation and Supercomputing in Nuclear Applications (M\&C + SNA 2007), Monterey, CA, USA, April 15-19, 2007.

[80] P. Girieud, "SCIENCE: The new FRAMATOME 3D nuclear code package for safety analysis," Proc. European Nucl. Conf. (ENC-94), Lyon, France, Oct. 2-6, 1994.

[81] V. Marotte, F. Clément, S. Thareau, S. Misu, I. Zmijarevic, "Industrial application of APOLLO2 to Boiling Water Reactors," Proc. Int. Conf. Advances in Nuclear Analysis and Simulation (PHYSOR 2006), Vancouver B.C., Canada, Sept. 10-14, 2006.

[82] J. Marten, F. Clément, V. Marotte, E. Martinolli, S. Misu, S. Thareau, L. Villatte, "The new AREVA NP spectral code APOLLO2-A,” Jahrestagung Kerntechnik 2007, Karlsruhe, Germany, 2007.

[83] E. Martinolli et al., "APOLLO2-A - AREVA's new generation lattice physics code: methodology and validation," Proc. Int. Conf. on Advances in Reactor Physics to Power the Nuclear Renaissance (PHYSOR 2010), Pittsburgh, PA, USA, May. 9-14, 2010.

[84] A. Pautz, H.W. Bolloni, K.A. Breith, R. van Geemert, J. Heinecke, G. Hobson, S. Merk, B. Pothet, F. Curca-Tivig, 
"The ARTEMIS core simulator: a central component in AREVA NP's code convergence project," Proc. Joint Int. Top. Mtg. on Mathematics \& Computation and Supercomputing in Nuclear Applications $(M \& C+S N A$ 2007), Monterey, CA, USA, April 15-19, 2007.

[85] S. Misu, H. Moon, "The SIEMENS 3-D steady state BWR core simulator MICROBURN-B2," Proc. Int. Mtg. on Physics of Nuclear Science and Technology, Long Island, NY, USA, Oct.5-8, 1998.

[86] "Introduction to HDF5 Data and Programming Models" in http://www.hdfgroup.org/pubs/presentations, 2008.

[87] "MCNP - A General Monte Carlo N-Particle Transport Code, Version 5," LANL, USA, 2004.

[88] S. Santandrea, "A new multi-domain DPN technique to accelerate the method of characteristics in unstructured meshes," Proc. Int. Top. Mtg. on Mathematics and Computation, Supercomputing, Reactor Physics and Nuclear and Biological Applications (M\&C2005), Avignon, France, Sept 12-15, 2005.

[89] I. Zmijarevic, "Multidimensional discrete ordinates nodal and characteristics methods for the APOLLO2 code," Proc. Int. Conf. on Mathematical Methods for Nuclear Applications,.Salt Lake City, UT, USA, Sept. 9-13, 2001.

[90] I. Zmijarevic, R. Sanchez, D. Lamponi, "Diffusion synthetic acceleration of Sn linear nodal schemes in weighted difference form," Proc. Int. Conf. on Mathematical Methods for Nuclear Applications, Salt Lake City, UT, USA, Sept. 9-13, 2001.

[91] L. Plagne and A. Ponçot, "Generic Programming for deterministic neutron transport codes," Proc. Int. Conf. on Mathematics and Computation, Supercomputing, Reactor Physics and Nuclear and biological Applications $(M \& C$ 2005), Avignon, France, Sept 12-15, 2005.

[92] J-F. Vidal, A. Calloo and P. Blaise, "Qualification of APOLLO2.8/JEFF-3.1.1 code package for the calculations of PWR plutonium recycling using the EPICURE experiments," Proc. Int. Conf. on Advances in Reactor Physics to Power the Nuclear Renaissance (PHYSOR 2010), Pittsburgh PA, USA, May 9-14, 2010.

[93] P. Blaise, O. Litaize, J-F. Vidal and A. Santamarina, "Qualification of the french APOLLO2.8/CEA2005v4 code package on absorber clusters in $17 \times 17$ PWR type lattices through the CAMELEON program," Proc. Int. Conf. on Advances in Reactor Physics to Power the Nuclear Renaissance (PHYSOR 2010), Pittsburgh PA, USA, May 9-14, 2010.

[94] J-F. Vidal, R. Le Tellier, P. Blaise, G. Guillot, N. Huot, O. Litaize, A. Santamarina, N. Thiollay and C. Vaglio-Gaudard, "Analysis of the FLUOLE experiment for the APOLLO2 validation of PWR core reflectors," Proc. Int. Conf. on Physics of Reactors, "Nuclear Power: A Sustainable Resource," (PHYSOR 2008), Interlaken, Switzerland, Sept. 14-19, 2008.

[95] C. Vaglio-Gaudard, A. Santamarina, M. El Hachmi and O. Litaize, "Accurate calculation of void reactivity in MOX lattice. Improvement of the APOLLO2 analysis of the $100 \%$ MOX MISTRAL3 experiment," Proc. Int. Conf. on Physics and Technology of Reactors and Applications (PHYTRA1), Marrakech, Morocco, March 14-16, 2007.

[96] T. Courau, M. Cometto, E. Girardi, D. Couyras and N. Schwartz, "Elements of Validation of Pin-by-Pin Calculations with the Future EDF Calculation Scheme Based on APOLLO2 and COCAGNE Codes", Proc. Int. Cong. on Advances in Nucl. Power Plants (ICAPP'08),
Anaheim, CA, USA, June 8-12, 2008.

[97] E. Girardi, M. Cometto, D. Couyras and N. Schwartz, "Pinby-Pin Power Reconstruction in the Future EDF Calculation Scheme", Proc. Int. Cong. on Advances in Nucl. Power Plants (ICAPP'08), Anaheim, CA, USA, June 8-12, 2008.

[98] F. Hoareau, N. Schwartz and D. Couyras, "A predictorcorrector scheme for the microscopic depletion solver of the COCAGNE core code," Proc. of the 18th Int. Conf. on Nuclear Engineering (ICONE18), Xi'an, China, May 17-21, 2010.

[99] J. Ragusa, R. Sanchez and S. Santandrea, "Application of duality principles to reflector homogenization," Nucl. Sci. Eng. 157, 299 (2007).

[100] C. Sandrin, R. Sanchez and F. Dolci, "An analysis of reflector homogenization techniques for full core diffusion calculations", Nucl. Sci. Eng. submitted June 2010.

[101] M. Grimod, R. Sanchez, F. Damian, "Neutronic Modeling for Pebble Bed Reactors," Proc. Int. Conf. on Mathematics, Computational Methods \& Reactor Physics (M\&C 2009), Saratoga Springs, NY, USA, May 3-7, 2009.

[102] E. Masiello, R. Clemente and S. Santandrea, "High Order Method of Characteristics for 2-D Unstructured Meshes," Proc. Int. Conf. on Mathematics, Computational Methods \& Reactor Physics (M\&C 2009), Saratoga Springs, NY, USA, May 3-7, 2009.

[103] G. Grassi, "A Nonlinear Space-Angle Multigrid Acceleration for the Method of Characteristics in Unstructured Meshes", Nucl. Sci. Eng. 155, 208 (2007).

[104] E. Masiello, "Analytical Stability Analysis of the CoarseMesh Finite Difference Method," Proc. Int. Conf. on Physics of Reactors, "Nuclear Power: A Sustainable Resource," (PHYSOR 2008), Interlaken, Switzerland, Sept. 14-19, 2008.

[105] R. Sanchez and J. Ragusa, "On the discrete-ordinates like collocation method and Galerkin angular quadratures. Part I.," Nucl. Sci. Eng. submitted May 2010.

[106] H. Golfier, R. Lenain, C. Calvin, J-J. Lautard, A-M. Baudron, P. Fougeras, P. Magat, E. Martinolli, Y. Dutheillet, "APOLLO3: a Common Project of CEA, AREVA and EDF for the Development of a New Deterministic Multi-purpose Code for Core Physics Analysis," Proc. Int. Conf. on Mathematics, Computational Methods \& Reactor Physics (M\&C 2009), Saratoga Springs, NY, USA, May 3-7, 2009.

[107] A.-M. Baudron and J.-J. Lautard, "SPN Core Calculations in the APOLLO3 System," Transp. Theory Stat. Physics, submitted June 2010.

[108] J.-J. Lautard, S. Loubière and C. Fedon-Magnaud, "CRONOS2 modular computational system for neutronic core calculations," Proc. of IAEA Specialist Mtg. on Advances Calculation Methods for Power Reactors, Cadarache, France, Sept. 10-14, 1990.

[109] G. Rimpault, D. Plisson, J. Tommasi, R. Jacqmin, J-M. Rieunier, D. Verrier and D. Biron, "The ERANOS Code and Data System for Fast Reactor Neutronic Analyses," Proc. Int. Conf. on Reactor Physics Safety and High-Performance Computing (PHYSOR 2002), Seoul, Korea, 7-10 October, 2002.

[110] A. Tsilanzara, Personal Communication, Commissariat à l'Energie Atomique, 2010.

[111] P. Mondot and R. Sanchez, "An iterative homogenization technique that preserves assembly core exchanges," Proc. Int. Conf. on Supercomputing in Nuclear Applications (SNA 2003), Paris, France, Sept. 22-24, 2003. 